This item was submitted to Loughborough's Research Repository by the author.

Items in Figshare are protected by copyright, with all rights reserved, unless otherwise indicated.

\title{
Direct current contact-mode triboelectric nanogenerators via systematic phase shifting
}

PLEASE CITE THE PUBLISHED VERSION

https://doi.org/10.1016/j.nanoen.2020.104887

\section{PUBLISHER}

Elsevier

\section{VERSION}

AM (Accepted Manuscript)

\section{PUBLISHER STATEMENT}

This paper was accepted for publication in the journal Nano Energy and the definitive published version is available at https://doi.org/10.1016/j.nanoen.2020.104887.

\section{LICENCE}

CC BY-NC-ND 4.0

\section{REPOSITORY RECORD}

Dharmasena, Ishara, HM Cronin, RA Dorey, and SRP Silva. 2020. "Direct Current Contact-mode Triboelectric Nanogenerators via Systematic Phase Shifting". Loughborough University. https://hdl.handle.net/2134/12486218.v1. 


\section{Direct Current Contact-Mode Triboelectric Nanogenerators via Systematic Phase Shifting}

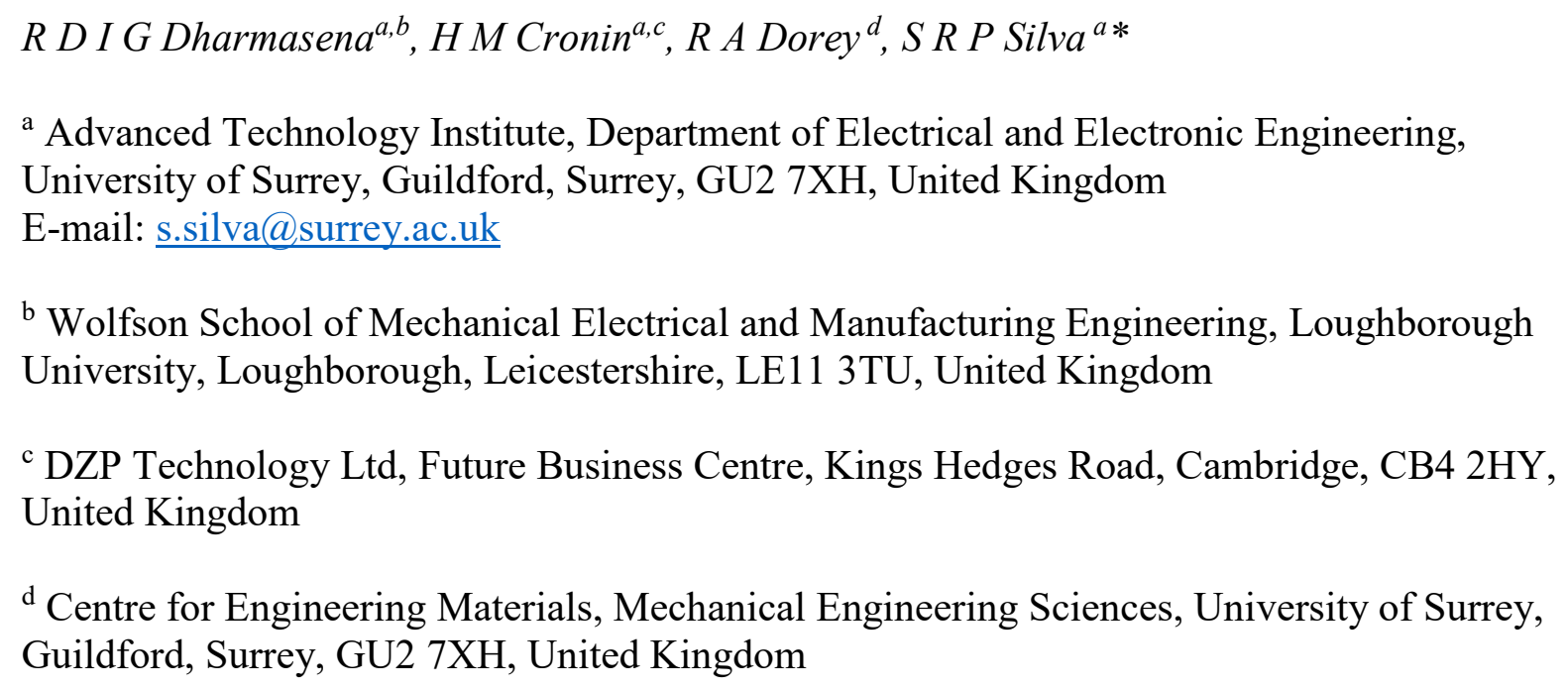

Abstract: The intermittency and discontinuous nature of power generation in Triboelectric Nanogenerators (TENGs) are arguably their most significant drawback, despite the promise demonstrated in low-power electronics. Herein, we introduce a novel technology to overcome this issue, in which, built-in systematic phase shifting of multiple poles is used to design a pseudo direct-current TENG. Unlike previous attempts of constructing near direct-current TENGs that base on the segmentation of electrodes of a sliding mode TENG, this technology introduces a new method that depends on planned excitation of constituent TENG units at different time intervals to obtain the necessary phase shifts, achieved by their structural design that contains an asymmetric spatial arrangement. Therefore, the direct current generation for

TENG, which was previously limited to the sliding mode TENG units, are expanded to contact-mode TENGs. The technology allows for continuous and smooth operation of the driven loads and paves the way for a new dawn in energy scavenging from mechanical sources. We use the distance-dependent electric field (DDEF) platform to design the systematic phase shifting technology, which is experimentally demonstrated via a freestanding mode TENG (FSTENG) based design, to power a number of prototype devices. The resultant power output of the TENG indicates a crest factor close to 1.1 at relatively low frequencies, the best reported values for TENGs with contact-mode basic units, to date. This 
work provides a highly awaited solution to overcome the intermittency and sporadic nature of

TENG outputs, thus, promoting the field towards powering next generation autonomous and mobile electronics.

\section{Graphical Abstract}

Triboelectric Nanogenerator is an emerging energy harvesting technology, however, the rapid variation of electrical outputs is seen as their most significant weakness. Herein, a novel technology to produce near direct current triboelectric nanogenerators is presented, applicable to a wide variety of device types and motion profiles.

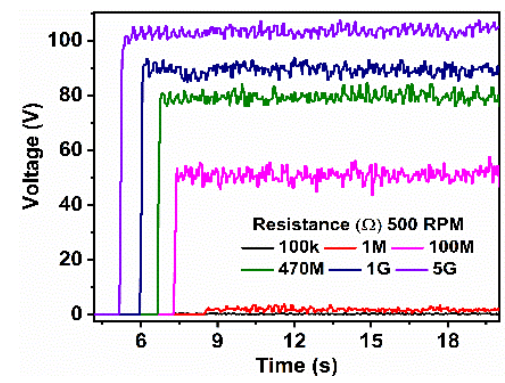

Keywords: energy harvesting, triboelectric nanogenerators, direct current nanogenerators, phase shift, distance dependent electric field model

\section{Introduction}

Recent developments in electronics technologies such as the internet of things (IoT), 5G technology are progressing at speed to revolutionise our lives ${ }^{[1-8]}$. Users and intelligent systems are connected with everyday objects through electronic elements such as sensors, displays and transducers, augmenting their functionality to construct a digitally connected network ${ }^{[3,7,9]}$. One of the key challenges here is to find suitable means of powering mobile, complex and scattered miniature electronic systems for autonomous operations ${ }^{[10,11]}$. Energy harvesting, which involves capturing energy from the ambient environment and converting 
into electricity, is widely considered as a viable option to power next generation electronic technologies ${ }^{[11,12]}$.

Triboelectric Nanogenerator (TENG), recently invented for mechanical energy harvesting, has been widely predicted to become a leading candidate for powering future low-power portable electronics ${ }^{[10,13-17]}$. In comparison to other energy harvesting technologies, TENGs offer a variety of advantages such as the low cost, high output power, high efficiency, ease of fabrication, flexibility, ergonomics and stretchability etc. ${ }^{[14,18-20]}$ The energy generation of TENGs depend on triboelectric effect, the separation of static charges due to the contact between two material surfaces, combined with the electrostatic induction resulting from the relative movement of such charged surfaces ${ }^{[10,21-23]}$. We recently presented the distancedependent electric field (DDEF) model to fully describe the working principles and output trends of TENG devices, and their output optimisation strategies ${ }^{[21,24-26]}$.

Despite significant improvements in the recent years, TENGs still need to overcome several inherent drawbacks to become a reliable power source for practical applications ${ }^{[10,14,15]}$. These devices produce instantaneous alternating current and voltage output pulses, due to the distance-dependent variation of their electric fields ${ }^{[21,24,25]}$. This causes a sporadic variation in the output power of TENGs, and, contradicts with the typical energy requirements of electronic devices ${ }^{[15]}$. Consequently, even when continuous mechanical energy is available, TENGs can only act as intermittent power sources ${ }^{[18]}$. Alternatively, power management circuits have been developed to modulate and store the output energy from the TENG ${ }^{[27-33]}$. While these designs enable desirable modification to the TENG outputs to an extent, they cannot typically eliminate the discontinuity, therefore, the continuous operation of the electronic applications is still disturbed. Additionally, the circuit elements used in these processes such as the transformers, transistors, inductors, capacitors etc. consume significant amounts of energy scavenged by the TENG, thus, drastically reducing the system efficiency $[25,31,34]$. 
The design and construction of direct current (DC) TENG devices, which produce and sustain near DC output signals, holds a paramount importance in improving the reliability, practicality and efficiency of triboelectric energy harvesting. Previous studies report attempts in producing DC TENGs through the use of bilaterally anchored electric brushes ${ }^{[35]}$. While the polarity change of the output was somewhat resolved, a large oscillation of the TENG output signals is observed in this approach. More recently, segmentation of electrodes has been used to obtain a near DC current output ${ }^{[34]}$. Furthermore, the triboelectric effect between liquid and dielectric surfaces has been used to produce a near DC TENG, which utilises the angular differences between multiple TENG units ${ }^{[36]}$. Typically, rotary movement is used in these device designs to continuously input mechanical energy into the system. ${ }^{[34-36]}$ Moreover, considering the motion of the triboelectrically charged surfaces, the predominant characteristic of almost all these designs is that the phase shift is achieved only when the triboelectric surfaces are moved in-plane, sliding with respect to each other. This limits the development of DC TENGs only to TENG architectures which utilise sliding movement between triboelectric layers to generate outputs (using sliding mode TENGs as their basic units). Therefore, almost all previous methods are compatible with only the In-plane charge polarization TENG (ICPTENG) based structures, whereas, there are limited known reports of DC TENG architectures developed with vertical charge polarization TENG (VCPTENG) as the basic unit. This greatly limits the applicability of DC TENG concept for a broad variety of applications as VCPTENG consists of the majority of highly efficient TENG architectures such as vertical contact separation mode TENG (VCSTENG) and free-standing mode TENG (FSTENG). ${ }^{[23]}$ Therefore, novel techniques in designing DC TENG architectures are urgently required to expand their applications.

In this work, following our DC TENG technology developed in early $2019^{[17,37]}$, we introduce a new technology to construct a DC TENG applicable for VCPTENGs, as well as for a range of motion profiles including rotary motion and linear oscillations. Herein, multiple TENG 
devices are used as poles of the TENG assembly, and the excitation of each of these TENG units are systematically controlled to obtain a phase shift between their outputs. This is achieved by engineering the spatial arrangement of the TENG units, and the structure of the TENG assembly. The phase shifted outputs from the TENG are then rectified and superimposed to obtain the resulting sustainable DC output. The distance-dependent electric field model is used to design and simulate the novel device structures. In the experimental demonstration, a TENG assembly consisting of multiple FSTENG units is developed. Following the characterisation of this device, the potential of this technology is demonstrated using a number of practical applications.

\section{Theory}

The output current of a TENG consists of positive and negative pulses, corresponding to ascending and descending motion cycles of its contact surfaces. The magnitude and the trends of this output depends on TENG material, device, and motion parameters, as well as the environmental factors, as discussed in the DDEF model from our previous work ${ }^{[10,21,24-26]}$. As a representative example, let's consider a vertical charge polarization TENG (VCPTENG) (device parameters detailed in Appendix 1), subjected to a single cycle of sinusoidal movement (1 mm amplitude, $1 \mathrm{~Hz}$ frequency). Considering the TENG output is extracted through an external load of $1 \mathrm{G} \Omega$, the experimental output current density (current per unit area) is depicted in Fig. 1a.

This behaviour can be modelled using the DDEF model, for further analysis. Figure $1 \mathrm{~b}$ depicts the DDEF model simulation for the same device, indicating the output current density through a $1 \mathrm{G} \Omega$ load plotted against time, for one contact-separation cycle. The current is composed of a positive peak and a negative peak corresponding to the contact and separation movement of TENG layers. Fig. 1c indicates the output trend when the TENG current is 
subjected to full wave rectification, resulting in two positive current peaks. However, a large variation in the current is clearly apparent within the output cycle for both Fig. 1b and Fig. 1c, which results in an exaggerated variation in output power (Fig. 1d). This variation was examined, and the device conditions were modified by incorporating an integrated phase shift mechanism to the TENG system, during this study.

The output from a number of TENG units can be integrated to enhance the total output current. As an example, the DDEF simulations for the overall output current obtained by adding the rectified current from six identical TENG units (similar to that described in Appendix 1) is depicted in Fig. 1e. Herein, the current outputs from the TENGs are assumed to be of the same phase, under identical motion cycles. Resultantly, the peak current values of each TENG add up to provide two distinct current peaks. Similarly, the instantaneous zero current positions line up, providing two zero current scenarios. The overall variation in the output current is high throughout the output cycle, similar to the single TENG unit. However, this variation can be minimized by systematically shifting the phase of the rectified output current from each of the TENG units with respect to each other, and, obtaining the overall superimposed output signal. This approach targets an even distribution of the current peaks of TENG units within the output cycle. For instance, considering the identical example of having six similar TENG units, a $60^{\circ}$ phase shift in the rectified current output between adjacent TENG units would provide a uniform distribution of current peaks within the output cycle (Fig. 1f). The overall output current in this scenario, obtained through the addition of these outputs on to common output terminal (superimposition) is depicted in Fig. 1g, which represents a relatively smooth near DC output current. The magnitude of the overall current is enhanced with respect to a case of a single TENG unit, due to the superposition of multiple output signals. Consequently, the power output of this TENG arrangement (Fig. 1h) is improved with respect to the single TENG unit shown in Fig. 1d, demonstrating significantly less variation within an output cycle. 
On the other hand, comparing the cases of the multiple TENG units with the similar phase and the phase shifted scenario, the output behaviour suggests that the overall energy generation in both these cases are similar. In the case of the multiple TENGs with similar phase, sharp and discontinuous output peaks are observed, whereas in the phase shifted scenario, the output peaks are distributed within the output cycle, resulting in a more regular uniform power output. Therefore, the phase shifted approach helps to reduce the fluctuation of the TENG output current via producing a near DC output.

The fluctuation in the output current of these TENG designs vary considerably depending on the number of TENG units (number of poles). Fig. 2 shows a theoretical analysis using the DDEF model on the variation of current and power outputs against different numbers of identical TENG units (identical to that described in Appendix 1, considering a 1 G $\Omega$ load), arranged to obtain systematic phase shifts between each other (under $1 \mathrm{~mm}$ amplitude and 1 $\mathrm{Hz}$ frequency). Fig. 2a indicates the current density of a single TENG unit compared to DC TENG architectures composed of 2 TENG units (with a phase angle of $180^{\circ}$ between their output current), 3 TENG units (with $120^{\circ}$ phase angle), 4 TENG units (with $90^{\circ}$ phase angle), 5 TENG units (with $72^{\circ}$ phase angle), and 6 TENG units (with $60^{\circ}$ phase angle), respectively. The current density increases significantly when the number of TENG units (poles) increase, as expected. Consequently, the power density increases in a concomitant manner, with higher numbers of TENG units providing significantly enhanced output power (Fig. 2b). The variation of the outputs is also reduced with the increasing number of TENG units, showing a behaviour closer to DC outputs (the example of an extended number of TENG units is demonstrated in Supplementary Note 2). Previous studies have utilised the crest factor; the ratio between the peak and the root mean square (RMS) of the output signal, as a measure of these variations. ${ }^{[34]} \mathrm{A}$ crest factor closer to 1 indicates a direct or a square waveform, whereas a higher crest factor represents a more fluctuating variation in the output behaviour. Fig. 2c shows the variation in the peak current and the RMS current, depicting the increase in both 
the values against the increasing number of TENG units. The difference between the two parameters decrease, as the number of TENG units increase. The smoothening of the output current is further confirmed by the decreasing crest factor against increasing number of TENG units (Fig. 2d). A similar trend can be observed for the power outputs, where the peak and RMS power densities increase (Fig. 2e) and the corresponding crest factor decrease (Fig. 2f), against increasing number of TENG units. The behaviour of the output voltage for this system, and the resultant crest factor, are depicted in Fig. $2 \mathrm{~g}$ and Fig. $2 \mathrm{~h}$ respectively, further confirming the trends observed in current and power output variations. Therefore, obtaining a systematic output phase shift between adjacent TENG units (poles) of a TENG assembly is a suitable method to develop a DC TENG energy harvester.

Obtaining these systematic phase shifts, thus far, have been limited to sliding-mode TENG units with segmented electrodes ${ }^{[34-36]}$, or, other comparable techniques ${ }^{[38][39]}$, which restricts the VCPTENGs from being utilised in DC TENG devices. In this work, we introduce a novel technique, which utilises the excitation of TENG units (poles) at different time intervals, to obtain a phase shift between them. This calculated excitation between different TENG units is achieved by an especially designed structure, in which they are arranged in an asymmetric geometry, providing the opportunity to use virtually any TENG architecture as the basic unit of the TENG assembly, along with the chance of using rotary or oscillating movements as the motion input.

\section{Experimental Design}

In order to experimentally realise the systematic excitation principle, a VCPTENG structure is used as a demonstrative example in this work. According to previous theoretical and experimental studies, the contact mode free-standing layer mode TENG (FSTENG), which belongs to VCPTENG category, is considered to be the most efficient working mode among 
all the major TENG working modes ${ }^{[24,40]}$. Therefore, FSTENG units were used as the basic energy generation units (poles) in the TENG architecture presented here (Fig. 3a). This was constructed in a cantilever arrangement, in which a polyethylene terephthalate (PET) sheet was coated with low density polyethylene (LDPE) to fabricate the free-standing layer (dimensions: $50 \mathrm{~mm} * 25 \mathrm{~mm} * 0.2 \mathrm{~mm}$ ). We highlight that, while other material combinations with higher triboelectric charging may be available, the material selection herein was purely based on the ease of fabrication and operation of the TENG units, targeting the proof of concept of the systematic excitation of TENGs. A pair of PET sheets $(35 \mathrm{~mm} * 25 \mathrm{~mm} * 0.2$ $\mathrm{mm}$ ) with a single sided $\mathrm{In}_{2} \mathrm{O}_{3} / \mathrm{Ag} / \mathrm{Au}$ conductive coating were used as the counter triboelectric surfaces, where the triboelectric charging was obtained from the contact between the PET and LDPE. The conductive coatings on the back sides of the PET sheets (counter triboelectric surfaces) acted as the electrodes for this device. It is important to notice that the mechanical excitation of the FSTENG was done by displacing the free-standing layer, which was fabricated to be longer than the counter triboelectric surfaces (Fig 3a). As depicted in Fig. 3a, separators were placed between the free-standing layer and the counter TENG layers to help gain adequate TENG layer separation following their contact. Electrical connectors were attached to the electrodes to extract the TENG outputs.

Multiple of such FSTENG units which act as the poles of the device (six for this example, following the DDEF model simulations) were combined to develop the integrated TENG assembly. A cylindrical holder (120 mm diameter, $60 \mathrm{~mm}$ height, $5 \mathrm{~mm}$ wall thickness) was 3D printed using polylactic acid (PLA) as the supporting structure for this architecture (Fig. 3b). In order to accommodate the FSTENG units, a set of openings were built into the cylindrical holder. Similarly, a rotor was fabricated with a cylindrical shaft (10 mm diameter, $60 \mathrm{~mm}$ height $)$ and six extended arms along its lower perimeter $(30 \mathrm{~mm} * 10 \mathrm{~mm} * 2 \mathrm{~mm})$ having even spacing between them (Fig. 3c). 
The integration of the FSTENG assembly was done in two different configurations. In the first case, which was used as the control architecture (referred to as the symmetric arrangement), the integrated TENG was constructed with FSTENG units placed inwards along the perimeter of the cylindrical holder, with equal gaps (marked as distance D) between adjacent units (Fig. 3d). When the TENG assembly is in operation, the rotor rotates due to the input motion, and the rotor arms (indicated as R1 to R6) strike against FSTENG units (marked as T1 to T6) providing the mechanical excitation for the free-standing layer of the FSTENG to oscillate. In this work, a small DC motor was used as the input motion source to the rotor, to simulate low rotational speed movements available in the environment, such as the movement of machines. The symmetry and the equal spacing of the architecture result in the contact between FSTENG and the extended arms occurring approximately at the same time for all six FSTENG units. The outputs of each FSTENG unit were fully rectified, and, supplied to common positive and negative output terminals (Fig. 3e) to obtain the overall superimposed output signal.

In the second configuration, the spacing between the FSTENG units was systematically changed to control the timing of their excitation in order to obtain a phase shift (referred to as the asymmetric arrangement) (Fig. 3f). To further explain this configuration, consider the position of the rotor shown in Fig. $3 \mathrm{f}$. In this arrangement, $\mathrm{R} 1$ and $\mathrm{T} 1$ contact first $\left(0^{\circ}\right.$ phase shift for the output current). In the previous case, the gap between T1 and T2 was D.

However, in the asymmetric TENG assembly, a systematic delay is created during the contact between $\mathrm{R} 2$ and $\mathrm{T} 2$ by introducing an additional $\mathrm{D} / 6$ gap for the position of $\mathrm{T} 2$, in comparison to where it would be placed in the symmetric scenario (gap between $\mathrm{T} 1$ and $\mathrm{T} 2$ is $\mathrm{D}+\mathrm{D} / 6$ for this case). This delays the contact between R2 and T2, therefore, the peak current positions of $\mathrm{T} 2$ are expected to shift by approximately $60^{\circ}$ in comparison to T1. A higher delay $\left(120^{\circ}\right.$ phase shift in the output current) is introduced for the contact between R3 and T3, by introducing 2D/6 additional gap for the position of T3, in comparison to the corresponding 
symmetric configuration. Similar incremental delay is introduced to the consecutive FSTENG units, where the maximum gap is observed for the contact between R6 and T6, with an additional gap of 5D/6 in comparison to the symmetric configuration, targeting a phase shift of $300^{\circ}$ in the output current generated by T6. Similar to that depicted in Fig. 3e, the outputs from each of the FSTENG units were rectified and supplied to common positive and negative terminals to obtain the overall output signal.

During the output characterisation, voltage outputs were measured using a Keithley 6517B Electrometer, whereas the current outputs were measured using a Keithley 6487 Picoammeter. The power outputs were measured based on the voltage outputs through different external loads. The experimental outputs were used to calculate the current and power generation per unit area (current density and power density), considering the overall triboelectrically active surface area (6 elements with $25 \mathrm{~mm} * 35 \mathrm{~mm}$ surface area) for each scenario.

\section{Results and Discussion}

The first step in the TENG characterisation was to observe the output trends of the basic power generation element; the FSTENG unit, which act as the poles of this device. During the operation of the FSTENG, triboelectric charging takes place on the two LDPE surfaces of the free-standing layer, and the two PET surfaces (Fig. 4a). The relative movement of the freestanding layer against the counter triboelectric layers causes an induced output current from the FSTENG. In the cantilever based FSTENG presented here, the extended arms of the rotor strike against the free-standing layer of the FSTENG unit (referred to as "flicking" action), causing it to oscillate several times (four to five cycles) after each contact. An alternating current is generated corresponding to this movement, consisting of multiple positive and negative peaks. To further study this behaviour, the short circuit current density $\left(J_{S C}\right)$ of a single FSTENG unit was measured before rectification (Fig. 4b). In this case, a single 
FSTENG unit was mounted on the cylindrical holder, and the rotor was operated at a relatively low speed of around $\sim 28$ revolutions per minute (RPM) to facilitate enhanced movement of a single FSTENG unit. The outputs demonstrated in Fig. $4 \mathrm{~b}$ shows that the flicking action of the cantilever based FSTENG unit increases its operating frequency specially at low rotational speeds. This is desirable for its power generation, since the increased frequency would result in increased power generation and reduced internal impedance, as demonstrated in our previous work ${ }^{[25]}$.

Following this, the integrated TENG assemblies were considered, one with the symmetric configuration (Fig. 3d) and the other with the asymmetric configuration (Fig. 3f). The outputs were rectified and connected to a common terminal for each case (Fig. 3e), which is referred to as the "overall current".

In order to obtain performance comparisons, the output current for both these cases were characterised at a relatively low rotational speed ( $28 \mathrm{RPM})$, as shown in Fig. $4 \mathrm{c}$. The overall current output of the symmetric arrangement appears to return to zero periodically, whereas a clear DC shift in the output signal exists for the asymmetric TENG arrangement. The minimum $J_{S C}$ of the asymmetric TENG is typically around $30 \mu \mathrm{A} / \mathrm{m}^{2}$, showing the potential of supplying a continuous non-zero output current for the contact mode TENG via systematic phase shifting, as predicted in the theory section. This proves the basic concept of having an engineered asymmetry in the TENG units, which creates a systematic excitation of each TENG at a specified time scale, hence, distributing the current peaks over the motion cycle of the device. This results in a novel method of constructing a DC TENG. After the proof of concept, a detailed analysis was conducted on the asymmetric TENG assembly, to further understand its output characteristics. The speed of the rotor was increased to 100 RPM, 250 RPM and 500 RPM, and the current (Fig. 4d-f) and voltage (Fig. 4g-i) were measured through a series of load resistors. At the beginning of the test, the rotor was kept turned off, and later turned on once the electrical measurement has started. It should be noted that the time axis for 
current and voltage has been stacked (shifted towards right side), and the current axis is represented in log scale, to ensure all outputs corresponding to all loads are shown on the same graph. The stacking of the outputs is done in the order of decreasing magnitude of outputs, so that the different states of the TENG outputs are clearly visible in Fig. 4d-i. The current outputs for all rotational speeds indicate continuous near DC characteristics. Considering the 100 RPM rotational speed (Fig. 4d), the output current is relatively high at low resistances $\left(\sim 170 \mu \mathrm{A} / \mathrm{m}^{2}\right.$ average current at $\left.100 \mathrm{k} \Omega\right)$, and, gradually decrease as the load resistance is increased $\left(\sim 4 \mu \mathrm{A} / \mathrm{m}^{2}\right.$ at $\left.5 \mathrm{G} \Omega\right)$. Similar reduction in the output under increasing load is observed for all rotational speeds. This current reduction against increasing load is consistent with the typical output behaviour observed for TENGs ${ }^{[21,24,25]}$, where the increasing external loads cause increased impedances towards the charge flow. On the other hand, increasing rotational speeds cause the current output to increase for a given load resistance. As an example, the average output current through $100 \mathrm{k} \Omega$ load increases from $\sim 170 \mu \mathrm{A} / \mathrm{m}^{2}$ for $100 \mathrm{RPM}$, to about $\sim 230 \mu \mathrm{A} / \mathrm{m}^{2}$ for $250 \mathrm{RPM}$, and, to around $\sim 380 \mu \mathrm{A} / \mathrm{m}^{2}$ for 500 RPM. A similar increment in output can be observed through the other load resistances, when the rotational speed is increased. Increased rotor speeds cause higher number of flicking cycles and excitation speeds of the FSTENGs at a given time period, which in turn causes larger output currents. This phenomenon is equivalent to the increased current outputs obtained using increased frequency of motion for VCPTENGs. ${ }^{[21,24,25]}$

Similarly, the output voltage was measured across the load resistor for voltage characterisations (Fig. 4g-i). Similar to the current outputs, the voltage values indicate a relatively stable trend without exaggerated oscillations, unlike the typical TENG voltage behaviour. The rotor is turned off initially, and the voltage increases when the rotor is turned on. At low load resistances, the output voltage is relatively low, which increases significantly when the load is increased. For example, considering 100 RPM case (Fig. 4g), the voltage is close to zero through $100 \mathrm{k} \Omega$, which increases close to $87 \mathrm{~V}$ through $5 \mathrm{G} \Omega$. The charge output 
passes readily through at low resistances, therefore, does not allow a potential difference to develop across the load, resulting in the observed behaviour. Similar to the current output trend, the voltage increases against increasing rotational speed. For instance, the average voltage output through $5 \mathrm{G} \Omega$ load increases from $\sim 87 \mathrm{~V}$ for $100 \mathrm{RPM}$ (Fig. $4 \mathrm{~g}$ ), to $\sim 95 \mathrm{~V}$ for 250 RPM (Fig. 4h), and to $\sim 103 \mathrm{~V}$ for 500 RPM (Fig. 4i). Increased rotor speed causes a higher current flow as discussed in the previous paragraph, which results in generating higher voltages for a given resistance. The crest factor for current (corresponding to current outputs in Fig. 4d-f) and voltage (based on voltage outputs in Fig. 4g-i) are shown in Fig. 4j and Fig. $4 \mathrm{k}$ respectively. As a general trend, the crest factor is relatively high at low resistances, and, decreases rapidly when the load is increased. Moreover, comparing the variations of the outputs at different rotational speeds, higher variations are generally observed at lower rotational speeds through most of the load resistances (Fig. 4j-k) (we note that minor deviations of this trend are visible via a minority of load resistances, potentially due to experimental and measurement variations). Consequently, lowest crest factors are observed at 500 RPM, indicating that the higher rotation speeds are capable of providing relatively smoother output signals. The lowest crest factors for current, voltage in this study are 1.17, 1.05 respectively, observed under 500 RPM rotational speed. These values provide strong evidence to the capability of the phase shifted asymmetric TENG architecture in producing near DC voltage and current outputs, demonstrating a significant improvement over previously reported TENG developments ${ }^{[34]}$.

Consequently, the resultant power output for the TENG assembly against time is depicted in Fig. 5a-c, at increasing external loads (100 k $\Omega$ to $5 \mathrm{G} \Omega$ ) and rotational speeds (100 RPM to 500 RPM). In this case, the power output is calculated based on the voltage measurements through increasing loads, and, the rotor is turned-on throughout the measurement. The DC nature of the power generation is noticeable across all rotational speeds and load resistances, providing a relatively stable and continuous power supply as opposed to intermittent power 
output pulses observed in conventional TENG architectures ${ }^{[14,19,34]}$. The average power output of the TENG against increasing load resistances is plotted for different rotational speeds in Fig. 5d. Increasing the speed of the rotor results in a significant improvement in the output power, for example, the maximum average power is around $0.36 \mathrm{~W} / \mathrm{m}^{2}$ at $100 \mathrm{RPM}$ which increases up to around $1 \mathrm{~W} / \mathrm{m}^{2}$ at $500 \mathrm{RPM}$. This behaviour is consistent with the predictions of the DDEF model ${ }^{[10,21]}$ and the TENG power transfer theory ${ }^{[25]}$, where increased rate of movement of TENG surfaces results in a higher rate of charge transfer between the electrodes, which in turn creates elevated currents, thus enhancing the output power. Furthermore, the output power varies significantly depending on the load resistance, similar to the typical TENG behaviour reported in previous studies. ${ }^{[10,21,24-26]}$ Considering the load resistances used in this study, the maximum power output for the TENG assembly is observed close to the 100 $\mathrm{M} \Omega$ load. Due to the increment of rotational speeds, a reduction in the load resistance corresponding to the maximum power generation (optimum load) is expected ${ }^{[25]}$, which is indicated in the trend lines in Fig. 5d. Considering the 100 RPM case, the optimum load is $\sim 120 \mathrm{M} \Omega$ load, and, according to the trend line corresponding to 500 RPM rotational speed, the optimum load is $\sim 50 \mathrm{M} \Omega$ to $60 \mathrm{M} \Omega$. Therefore, the trend lines clearly suggest an anticipated reduction in the optimum load resistance as the rotational speed is increased. This is again consistent with the DDEF model simulations and TENG power transfer theory, as the elevated rate of movement of TENG layers effectively reduces the device impedance. During the maximum power output condition, the external load matches the device impedance, therefore, resulting in the observed reduction in the optimum load resistance.

The crest factor corresponding to power outputs (based on power outputs in Fig. 5a-c) is shown in Fig. 5e. The trends of power crest factor are similar to the voltage and current crest factors, which decreases against increased load resistances and increased rotational speeds. The lowest crest factors power is 1.08 , again observed under 500 RPM rotational speed. The power output behaviour and the low power crest factor indicates the enhanced capability of 
the phase shifted asymmetric TENG architecture in producing near DC power outputs, demonstrating significant improvements in the area of DC TENGs.

The outputs of the DC TENG assembly was used to demonstrate the capability of continuously powering practical applications. In the first demonstration, the DC TENG assembly was connected directly to a series of LED lights at a rotor speed of 250 RPM. As shown in Fig. 6a and supplementary video 1, multiple LED lights were continuously powered, depicting a significant enhancement of power generation characteristics over conventional contact mode TENG devices. Secondly, a bespoke photodetector was fabricated by depositing a photoactive perovskite ink on interdigitated electrodes, on a flexible PET substrate, and, the DC TENG was used to continuously power this photodetector. Fig. $6 \mathrm{~b}$ depicts the output signal detected from the photodetector when subjected to the visible light from a solar simulator at an intensity of $100 \mathrm{~mW} / \mathrm{m}^{2}$, where a clear step change in the output signal is detected when the light is turned on. Therefore, the contact mode DC TENG introduced in this work demonstrates the potential of providing a near DC output, significantly widening the range of future applications for triboelectric energy harvesting.

\section{Future Perspectives and Outlook}

The asymmetric spatial arrangement of TENG poles to obtain the phase shift for a DC TENG via engineered excitation was introduced for rotary movements and VCPTENG device architectures in the previous section. Similarly, the applicability of this principle was investigated against linear oscillations. For example, in the TENG assembly depicted in Fig. 7a, VCPTENG units (poles) similar to that discussed in section 3 are arranged with same gap distances (D) between them. A similar number of excitation units (arms) are placed with increasing distances between them to obtain the systematic excitation of TENG units (eg: distance between $\mathrm{R} 1$ and $\mathrm{R} 2$ is $\mathrm{D}+\mathrm{D} / 6$ etc.), equivalent to the gaps between the TENG units shown in Fig. 3d. The oscillation unit is connected to the mechanical energy source (ex: 
vibration energy source such as a machine surface or a piston), and assuming the position given in Fig. 7a and the oscillation unit is moving rightwards, R6 will contact T6 first. This will be followed by the contact between R5 and T5 etc., whereas the contact between R1 and T1 will take place at the end. The outputs are rectified and connected to a common terminal, as depicted in Fig. 7 a.

The DDEF model was used to simulate the output trends of this device, assuming typical operating conditions of $5 \mathrm{~mm}$ amplitude and $5 \mathrm{~Hz}$ frequency, while the other parameters are identical to that described in the Theory section. As indicated in Fig. 7b and Fig. 7c, both the current density and power density indicate a near DC behaviour with their average output densities reaching $560 \mu \mathrm{A} / \mathrm{m}^{2}$ and $317 \mathrm{~W} / \mathrm{m}^{2}$, along with crest factors of 1.08 and 1.17 respectively, providing evidence for the applicability of this concept to linear oscillation movements.

Herein we also note that, the systematic phase shifting technique presented in this work is significantly different from the work presented very recently by Li et al. ${ }^{[41]}$ Firstly, the phase shifting concept presented here is based on the asymmetric spatial arrangement of TENG units and their systematic excitation as demonstrated by the DDEF model simulations (Fig. 1) and the experimental setup (Fig. 3), compared to the symmetric arrangement of the aforementioned study. The effectiveness of our method is highlighted in the initial experimental (Fig. 4c) and theoretical results (Fig. 1d, h). Moreover, using this technique, we were able to obtain significantly low current crest factors (close to 1.1) using notably less number of TENG poles (6), compared to the comparatively high crest factor (1.31) obtained previously using a larger number of TENG poles $(24)^{[41]}$. Finally, as depicted earlier in this section, we have demonstrated our concept for both rotary movements as well as linear oscillations, including VCPTENG devices, which has not been reported previously. Furthermore, we note that the performance characteristics of the DC TENG structures presented here can be improved by enhancing the performance of the individual TENG poles. 
As we demonstrated in our previous work ${ }^{[25]}$ increasing the surface area of the contact surfaces, increasing the number of TENG poles, increasing the surface charge density of the TENG, reducing the thickness of TENG sheets, increasing the motion rate and engineering the dielectric constant can potentially be used to increase the output power density and reduce the internal impedance of the TENG poles, hence, of the DC TENG device. Moreover, as demonstrated in this work, using larger number of TENG poles would generate more smooth DC output signals, resulting in further reduction in the crest factor. These performance enhancement techniques and optimisation methods need to be investigated with respect to the DC TENG, which would increase its capability of powering real time practical applications.

\section{Conclusions}

This work presents a novel method of realising a DC TENG, by shifting the phase of a series of built in TENG units which act as the poles of the device. The constituent poles (TENG units) are excited at calculated time intervals in order to obtain the required phase shift between their outputs, which is achieved by designing their spatial arrangement. The output of each pole is rectified and added to a common pair of output terminals, resulting in the DC outputs. Unlike all previous methods in constructing DC TENGs which are limited only to inplane charge polarization TENGs with rotary motion, this new method is compatible with VCPTENG units used as poles, as well as with rotary and linear oscillation motion types. The DDEF model is used as the basis of deriving the theoretical outputs and designing the TENG architectures. As demonstrated in the experimental results for the FSTENG based rotary type device, overall outputs of the DC TENG demonstrate near DC characteristics, overcoming many of the intermittency and inconsistency issues associated with conventional TENG designs. Increasing the number of TENG units and rate of excitation (up to 6 TENG units and 500 RPM investigated in this study) results in enhanced performance of the DC TENG. The experimental analysis indicates a crest factor close to 1.1 for the power output of 
the DC TENG, resulting in a significant reduction in the sporadic variation of power output normally associated with TENGs. Potential of the DC TENG in powering electronic components is successfully demonstrated by using its output to continuously operate a series of LEDs and a bespoke flexible photo detector.

Similarly, a FSTENG based DC TENG which operates using linear oscillation movements is demonstrated using the DDEF model, indicating current and power crest factors of 1.08 and 1.17 at an oscillation frequency of $5 \mathrm{~Hz}$, relatively close to the ideal value of 1.0 .

Therefore, this work presents a novel technology which addresses one of the key bottlenecks of mechanical energy harvesting, paving the path towards the successful development of sustainable practical applications.

\section{Supporting Information}

Supporting Information is included as the supplementary note 1, and, the supplementary video 1.

\section{Author Contributions}

R.D.I.G.D. invented, designed and conducted the theoretical and experimental work. S.R.P.S. supervised the project. R.A.D. supported the 3D printing of the TENG and H.M.C. supported the photodetector fabrication. R.D.I.G.D. and S.R.P.S. wrote the manuscript and organised the figures. All authors discussed the results and commented on the manuscript.

\section{Acknowledgements}

The authors would like to acknowledge the support from the EPSRC research project grant EP/S02106X/1 in providing the funding for this work. R.D.I.G.D. acknowledges the support from the Loughborough University Doctoral Prize Fellowship. H.M.C. acknowledges the Royal Commission for the Exhibition of 1851 for their support. 


\section{Competing Interests}

R.D.I.G.D. and S.R.P.S. are the inventors of the patent application with the title "Triboelectric Generator" under GB priority application no: GB1819309.4. The other authors do not declare conflicts of interest.

\section{References}

[1] R. D. I. G. Dharmasena, K. D. G. I. Jayawardena, Z. Saadi, X. Yao, R. M. I. Bandara, Y. Zhao, S. R. P. Silva, Proc. IEEE 2019, 1.

[2] J. Gubbi, R. Buyya, S. Marusic, M. Palaniswami, Futur. Gener. Comput. Syst. 2013, $29,1645$.

[3] D. Singh, G. Tripathi, A. J. Jara, in 2014 IEEE World Forum Internet Things, IEEE, 2014, pp. 287-292.

[4] M. Stoppa, A. Chiolerio, Sensors 2014, 14, DOI 10.3390/s140711957.

[5] K. E. Skouby, P. Lynggaard, in 2014 Int. Conf. Contemp. Comput. Informatics, IEEE, 2014, pp. 874-878.

[6] I. A. T. Hashem, V. Chang, N. B. Anuar, K. Adewole, I. Yaqoob, A. Gani, E. Ahmed, H. Chiroma, Int. J. Inf. Manage. 2016, 36, 748.

[7] S. Li, L. Da Xu, S. Zhao, J. Ind. Inf. Integr. 2018, 10, 1.

[8] R. N. Wijesena, P. N. D. Tissera, K. M. N. de Silva, G. A. J. Amaratunga, R. D. I. G. Dharmasena, W. R. L. N. Bandara, A. K. L. A. Wijenayaka, R. M. I. Bandara, Near Infrared Energy Absorbing Textile, 2016, US20170314185A1.

[9] W. Weng, P. Chen, S. He, X. Sun, H. Peng, Angew. Chem. Int. Ed. Engl. 2016, 55, 6140.

[10] R. D. I. G. Dharmasena, S. R. P. Silva, Nano Energy 2019, 62, 530.

[11] P. Kamalinejad, C. Mahapatra, Z. Sheng, S. Mirabbasi, V. C. M. Leung, Y. L. Guan, IEEE Commun. Mag. 2015, 53, 102.

[12] H. G. Lee, N. Chang, in 20th Asia South Pacific Des. Autom. Conf., IEEE, 2015, pp. $124-129$. 
[13] F. R. Fan, Z. Q. Tian, Z. Lin Wang, Nano Energy 2012, 1, 328.

[14] Z. L. Wang, Faraday Discuss. 2015, 176, 447.

[15] C. Wu, A. C. Wang, W. Ding, H. Guo, Z. L. Wang, Adv. Energy Mater. 2019, 9, 1802906.

[16] K. Zhao, G. Gu, Y. Zhang, B. Zhang, F. Yang, L. Zhao, M. Zheng, G. Cheng, Z. Du, Nano Energy 2018, 53, 898 .

[17] R. D. I. G. Dharmasena, Triboelectric Self-Powered Energy Systems. [Doctoral Dissertation], University of Surrey, 2019.

[18] F. R. Fan, W. Tang, Z. L. Wang, Adv. Mater. 2016, 28, 4283.

[19] J. Chen, Z. L. Wang, Joule 2017, 1, 480.

[20] Y. Wang, Y. Yang, Z. L. Wang, npj Flex. Electron. 2017, 1, 10.

[21] R. D. I. G. Dharmasena, K. D. G. I. Jayawardena, C. A. Mills, J. H. B. Deane, J. V. Anguita, R. A. Dorey, S. R. P. Silva, Energy Environ. Sci. 2017, 10, 1801.

[22] S. Niu, Z. L. Wang, Nano Energy 2015, 14, 161.

[23] S. Niu, S. Wang, L. Lin, Y. Liu, Y. S. Zhou, Y. Hu, Z. L. Wang, Energy Environ. Sci. 2013, 6, 3576 .

[24] R. D. I. G. Dharmasena, K. D. G. I. Jayawardena, C. A. Mills, R. A. Dorey, S. R. P. Silva, Nano Energy 2018, 48, 391.

[25] R. D. I. G. Dharmasena, J. H. B. Deane, S. R. P. Silva, Adv. Energy Mater. 2018, 1802190.

[26] J. H. B. Deane, R. D. I. G. Dharmasena, G. Gentile, Nano Energy 2018, 54, 39.

[27] F. Xi, Y. Pang, W. Li, T. Jiang, L. Zhang, T. Guo, G. Liu, C. Zhang, Z. L. Wang, Nano Energy 2017, 37, 168.

[28] D. Bao, L. Luo, Z. Zhang, T. Ren, J. Semicond. 2017, 38, 095001.

[29] H. Qin, G. Cheng, Y. Zi, G. Gu, B. Zhang, W. Shang, F. Yang, J. Yang, Z. Du, Z. L. Wang, Adv. Funct. Mater. 2018, 28, 1805216. 
[30] X. Liang, T. Jiang, G. Liu, T. Xiao, L. Xu, W. Li, F. Xi, C. Zhang, Z. L. Wang, Adv. Funct. Mater. 2019, 1807241.

[31] Y. Zi, H. Guo, J. Wang, Z. Wen, S. Li, C. Hu, Z. L. Wang, Nano Energy 2017, 31, 302.

[32] G. Cheng, H. Zheng, F. Yang, L. Zhao, M. Zheng, J. Yang, H. Qin, Z. Du, Z. L. Wang, Nano Energy 2018, 44, 208.

[33] H. Qin, G. Gu, W. Shang, H. Luo, W. Zhang, P. Cui, B. Zhang, J. Guo, G. Cheng, Z. Du, Nano Energy 2020, 68, 104372.

[34] H. Ryu, J. H. Lee, U. Khan, S. S. Kwak, R. Hinchet, S.-W. Kim, Energy Environ. Sci. 2018, DOI 10.1039/C8EE00188J.

[35] J. Wang, Z. Wu, L. Pan, R. Gao, B. Zhang, L. Yang, H. Guo, R. Liao, Z. L. Wang, ACS Nano 2019, acsnano.8b09642.

[36] T. Kim, D. Y. Kim, J. Yun, B. Kim, S. H. Lee, D. Kim, S. Lee, Nano Energy 2018, 52, 95.

[37] R. D. I. G. Dharmasena, S. R. P. Silva, Triboelectric Generator, 2019, United Kingdom Patent Application No. GB2019/053344.

[38] D. Liu, X. Yin, H. Guo, L. Zhou, X. Li, C. Zhang, J. Wang, Z. L. Wang, Sci. Adv. 2019, 5, DOI 10.1126/sciadv.aav6437.

[39] R. Xu, Q. Zhang, J. Y. Wang, D. Liu, J. Wang, Z. L. Wang, Nano Energy 2019, DOI 10.1016/j.nanoen.2019.104185.

[40] Y. Zi, S. Niu, J. Wang, Z. Wen, W. Tang, Z. L. Wang, Nat. Commun. 2015, 6, 8376.

[41] X. Li, X. Yin, Z. Zhao, L. Zhou, D. Liu, C. Zhang, C. Zhang, W. Zhang, S. Li, J. Wang, Z. L. Wang, Adv. Energy Mater. 2020, 10, 1903024. 
Figures

(a)

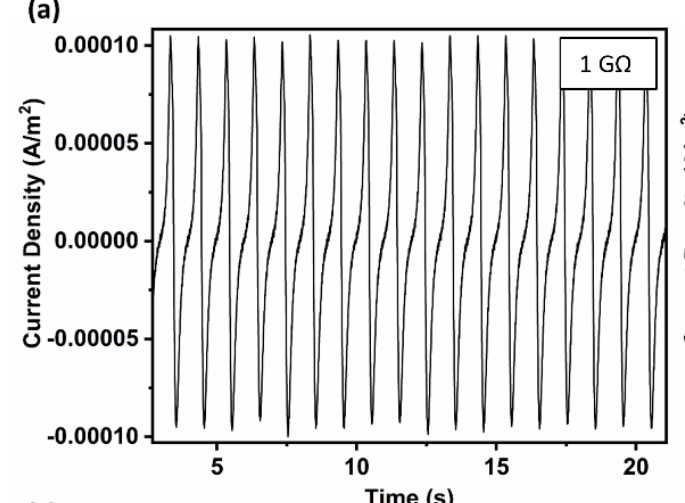

(c)

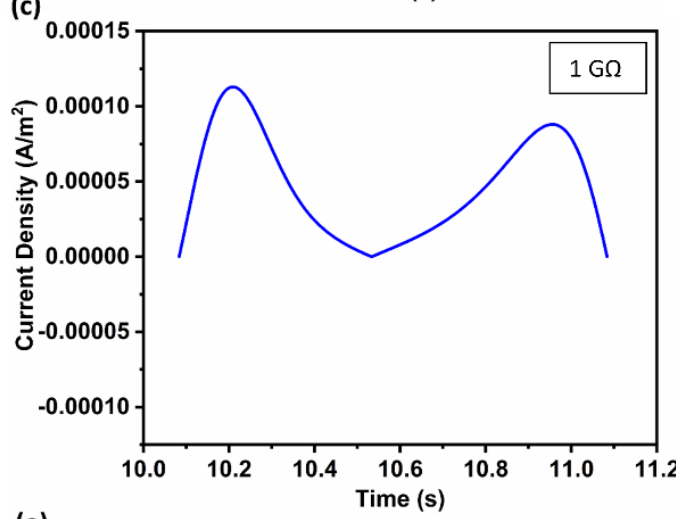

(e)
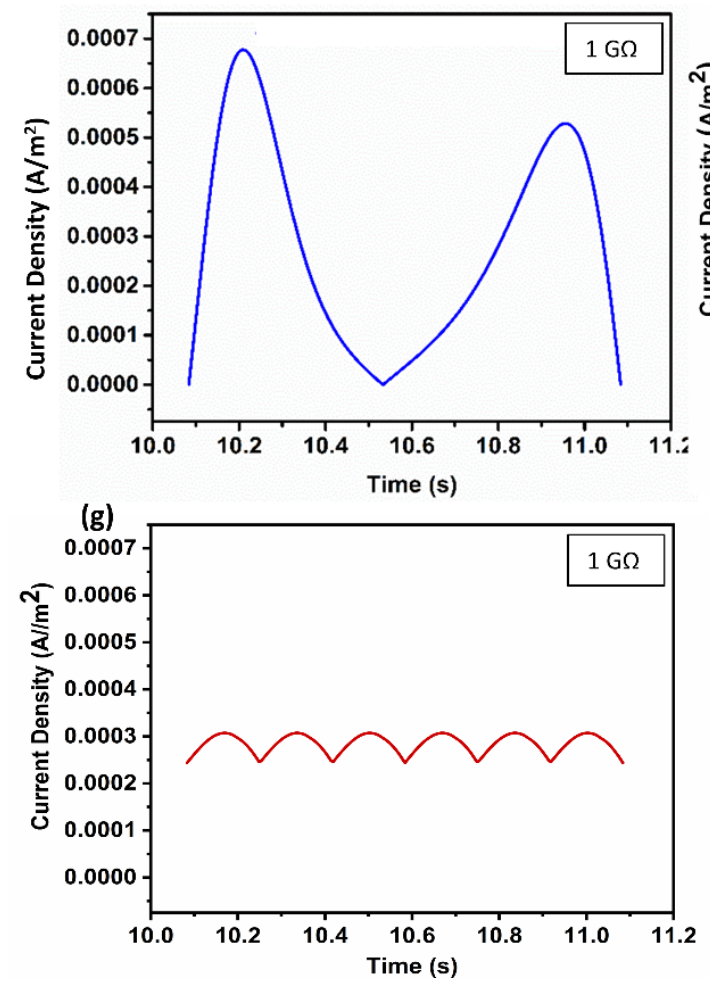

(b)

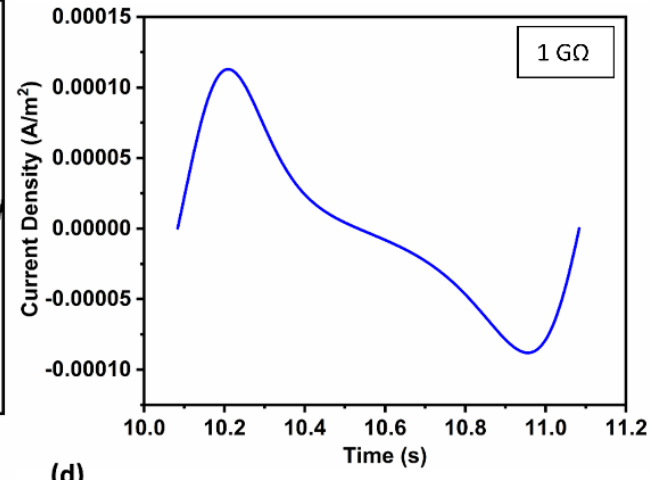

(d)

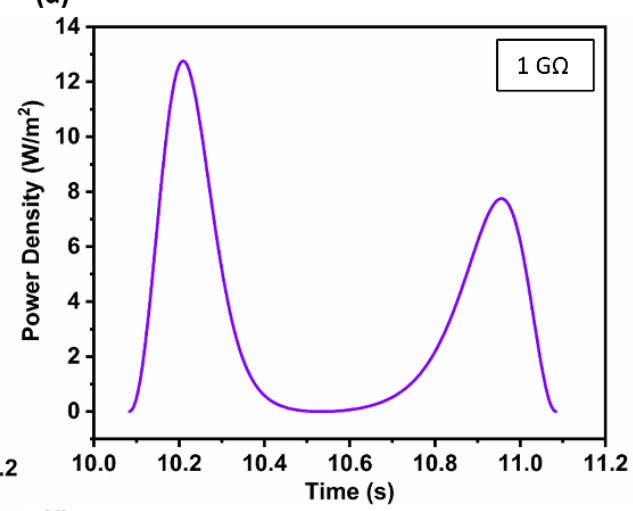

(f)

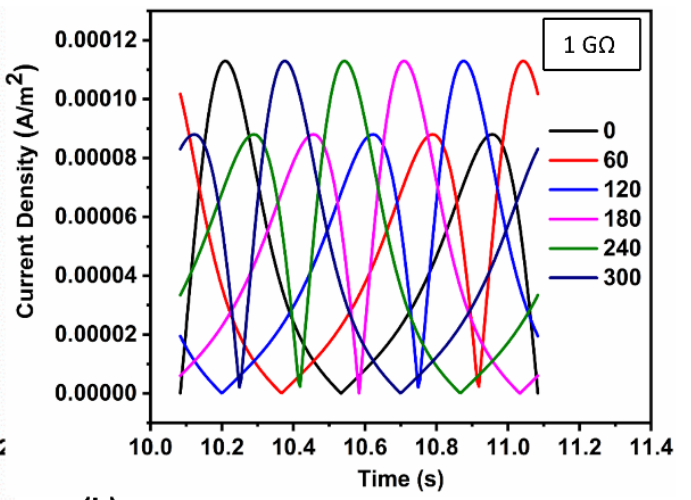

(h)

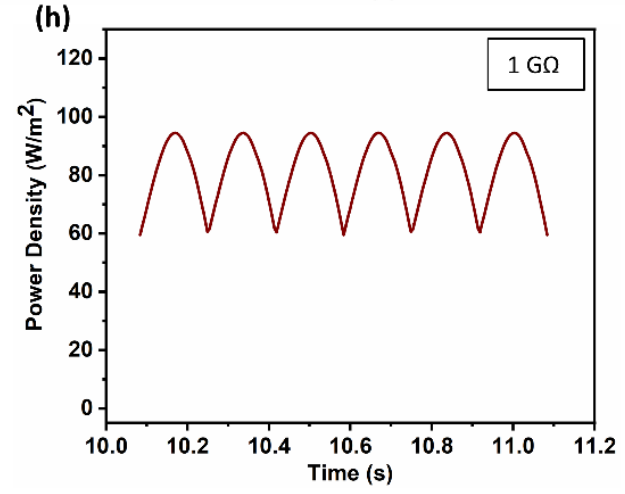

Figure 1: (a) The experimental current output (per unit area) of a VCPTENG (as described in Appendix 1) through a $1 \mathrm{G} \Omega$ load, subject to $1 \mathrm{~mm}$ amplitude and $1 \mathrm{~Hz}$ frequency sinusoidal movement. (b) The distance-dependent electric field (DDEF) model simulations for the current output (normalised per unit area) of a TENG similar to that in (a) under identical working conditions, depicting a single contact-separation cycle. (c) The current output of the TENG 
described in (b), when subjected to full wave rectification, and, (d) the resultant power output. (e) The rectified and integrated current output of six TENG devices similar to that in (b) which are in same phase. (f) A demonstration of the overlap between the current signals of six TENG units similar to (e), with a systematic phase shift of $60^{\circ}$ between the adjacent TENGs. The overall $(\mathrm{g})$ current output, and, (h) power output resulting from the TENG arrangement with a systematic phase shift, described in (f).

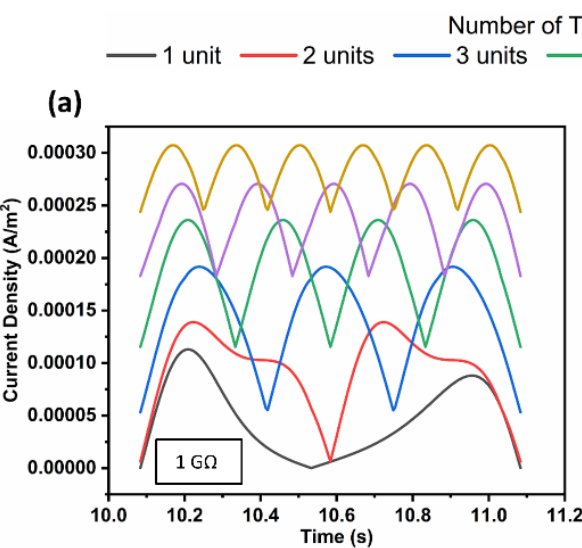

(c)

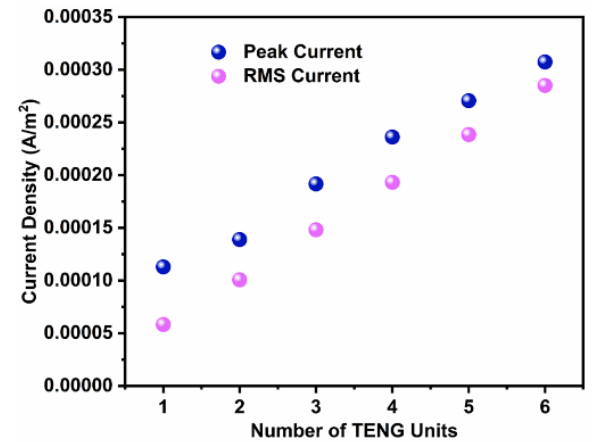

(d)

(e)

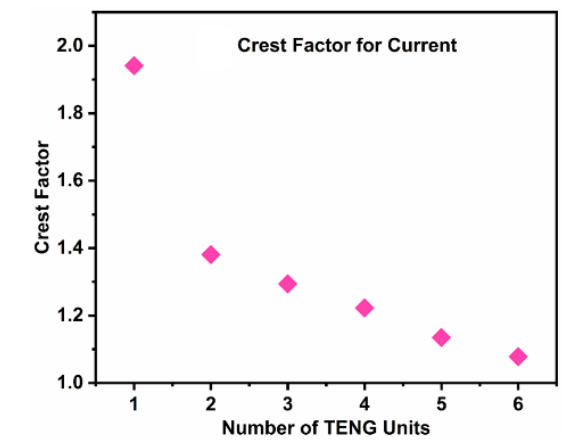

(f)
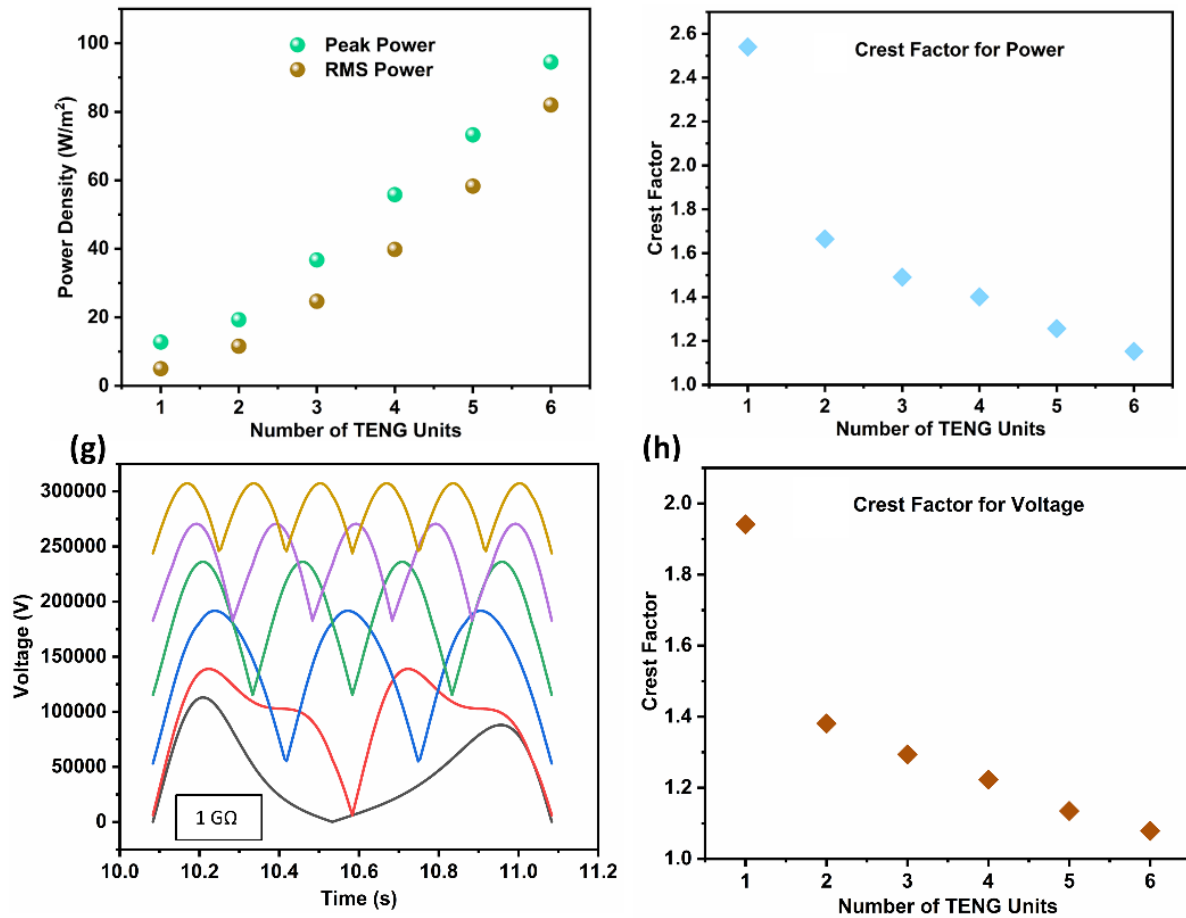

(h)
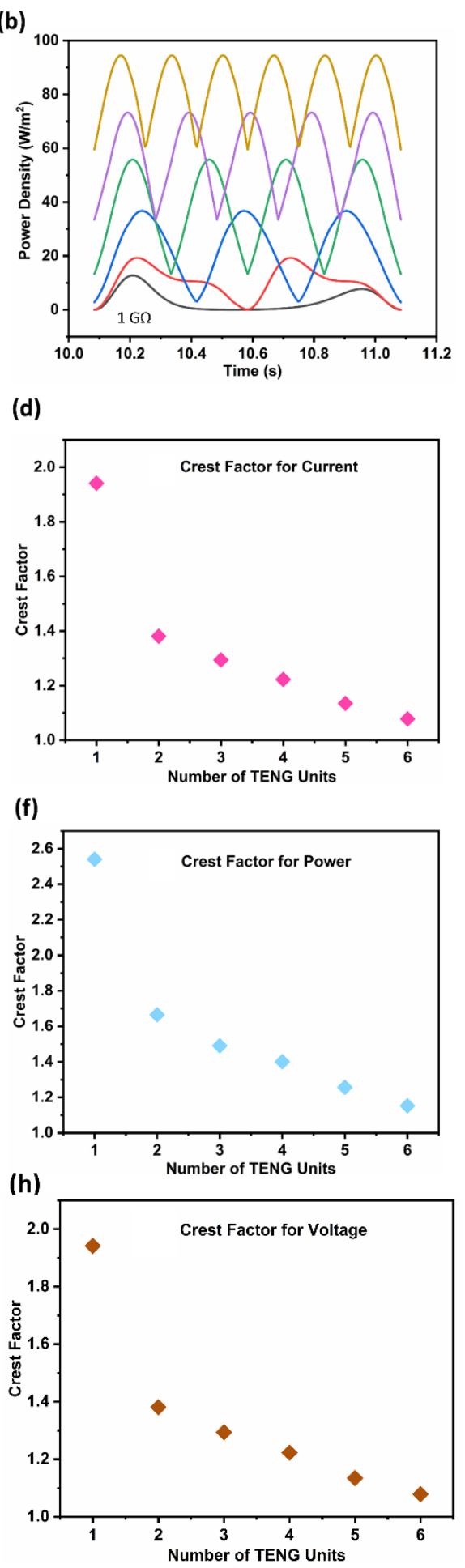
Figure 2: The variation of the (a) Current density, and, (b) Power density (through a 1 G $\Omega$ load) for TENG architectures with increasing number of TENG units (identical to that described in Appendix 1), subjected to $1 \mathrm{~mm}$ amplitude and $1 \mathrm{~Hz}$ frequency sinusoidal movement. (c) The peak current and RMS current density values corresponding to (a), plotted against the number of TENG units, and, (d) resultant crest factors. (e) The peak and RMS power density values corresponding to (b), plotted against the number of TENG units, and, (f) resultant crest factors. (g) Variation of the voltage for the same arrangement described in (a), and, (h) the resultant crest factor considering the voltage outputs.

(a)

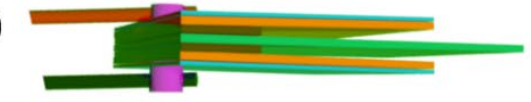

PET covered with LDPE (free standing TENG layer)

PET (counter TENG layers) $\operatorname{In}_{2} \mathrm{O}_{3} / \mathrm{Ag} / \mathrm{Au}$ coating (electrodes)

Separators

Output lead 1

(b)

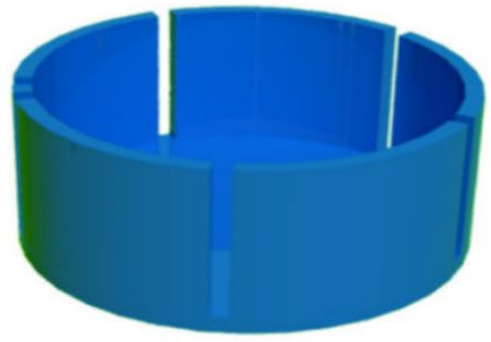

(d)
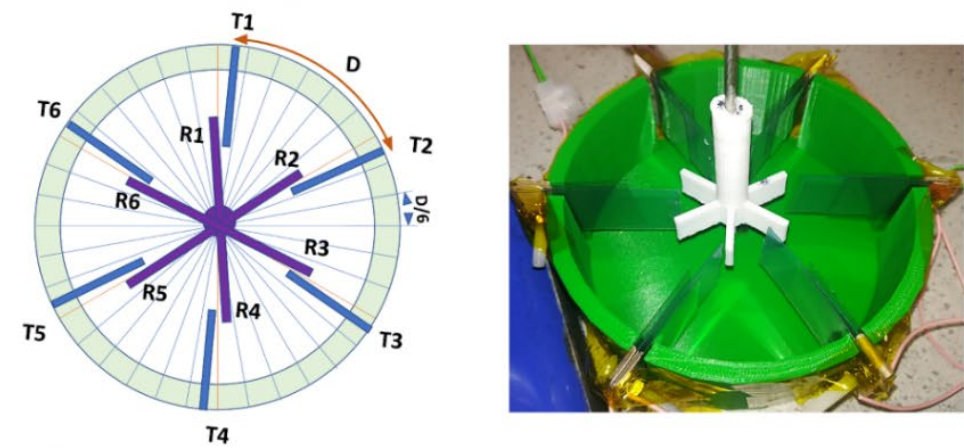

TENG Units (T1 to T6)

(e)

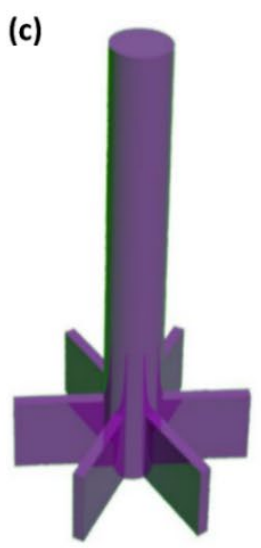


Figure 3: (a) Schematic of the structure of the FSTENG unit, which is used as the basic energy generation element of the DC TENG. (b) The cylindrical supporting structure of the TENG arrangement. (c) The rotor with extended arms to provide the motion input for the TENGs. (d) Schematic (left) and the photograph (right) of the symmetric TENG assembly, designed to obtain a current output comparable to Fig. 1e. (e) Schematic of the full wave rectification of the output from each TENG unit, and the integration of overall output into $(+)$ and $(-)$ terminals. (f) Schematic (left) and the photograph (right) of the asymmetric TENG assembly, designed to obtain a systematic phase shift in the output of TENG units, by changing their gap distances. 
(a)

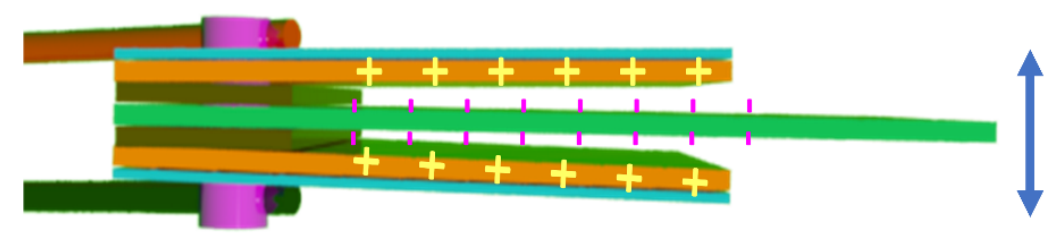

(b)

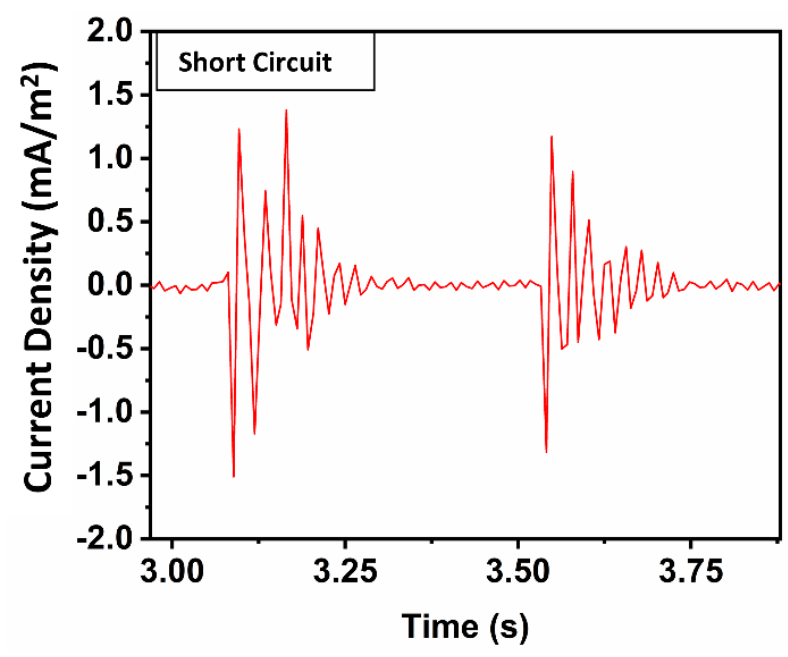

(d)
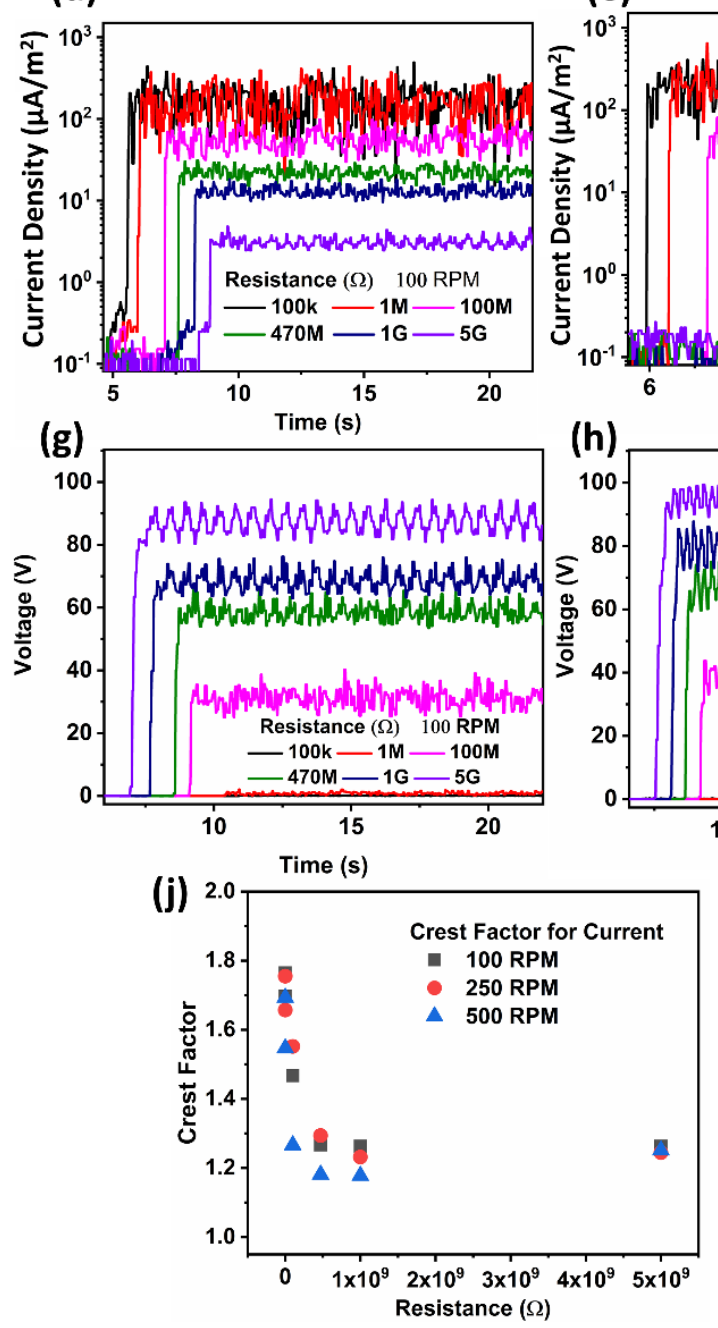

(c)

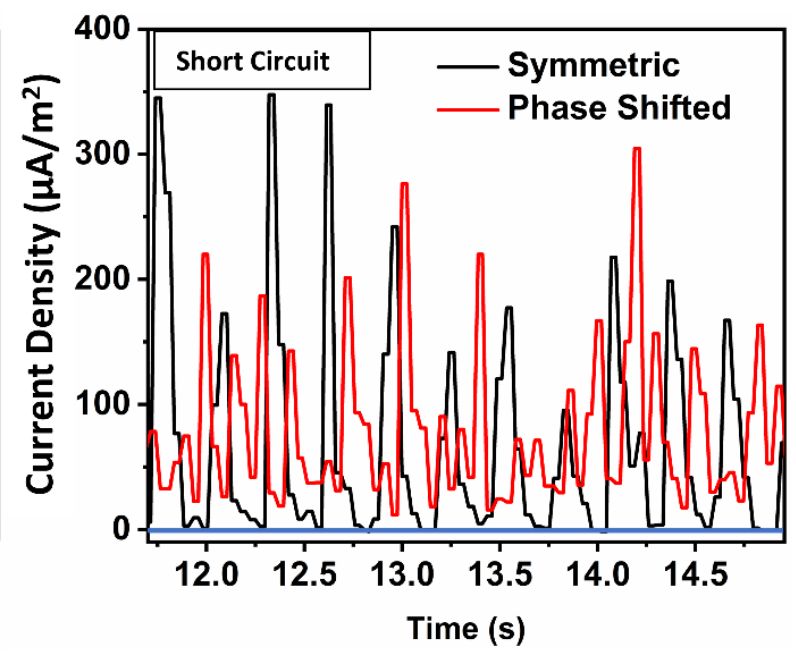

(f)
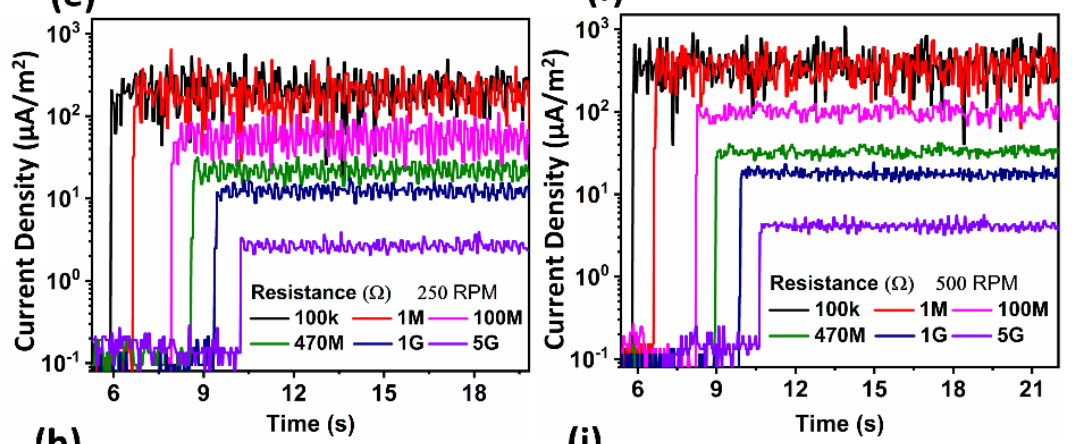

(i)
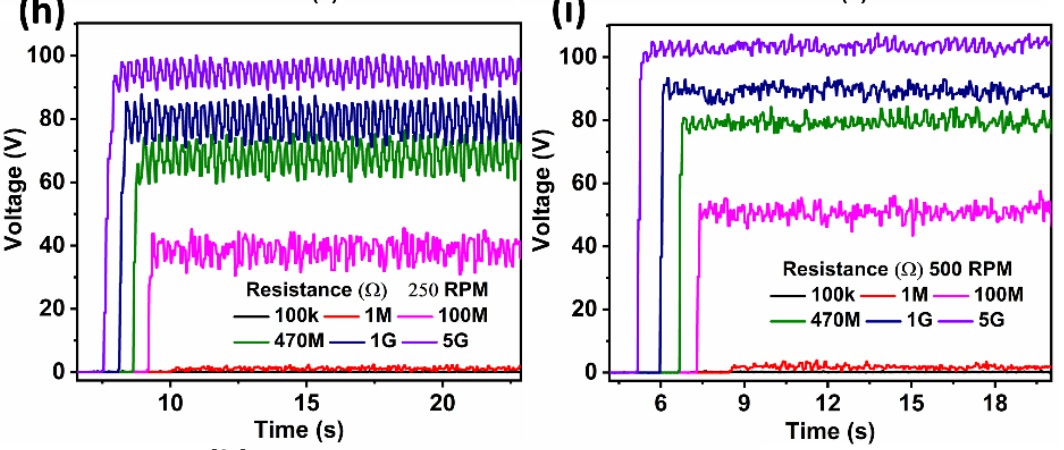

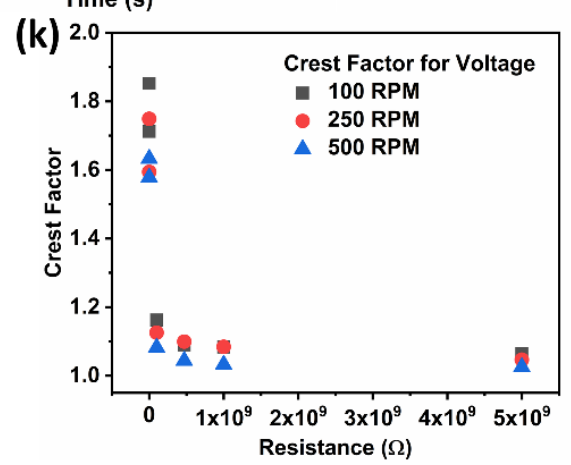

Figure 4: (a) Schematic representation of the triboelectric charging of FSTENG unit, which is used as the basic power generation unit of the DC TENG. The components of the FSTENG is 
identical to that depicted in Fig. 3a. (b) $J_{S C}$ of a FSTENG unit during two consecutive flicks between the rotor and the free-standing layer, demonstrating the increase in the operating frequency (multiple current peaks during each flick). (c) A comparison between the $J_{S C}$ of the symmetric configuration (Fig. 3d) and the asymmetric configuration (Fig. 3f) of the TENG assembly, at a rotor speed of 28 RPM. Current output (per unit area) of the asymmetric TENG architecture through increasing loads, at (d) 100 RPM, (e) 250 RPM, and, (f) 500 RPM rotor speeds. Voltage output of the asymmetric TENG architecture through increasing loads, at (g) 100 RPM, (h) 250 RPM, and, (i) 500 RPM rotor speeds. The corresponding crest factors for (j) current, and, (k) voltage outputs of the asymmetric TENG, plotted against increasing loads.
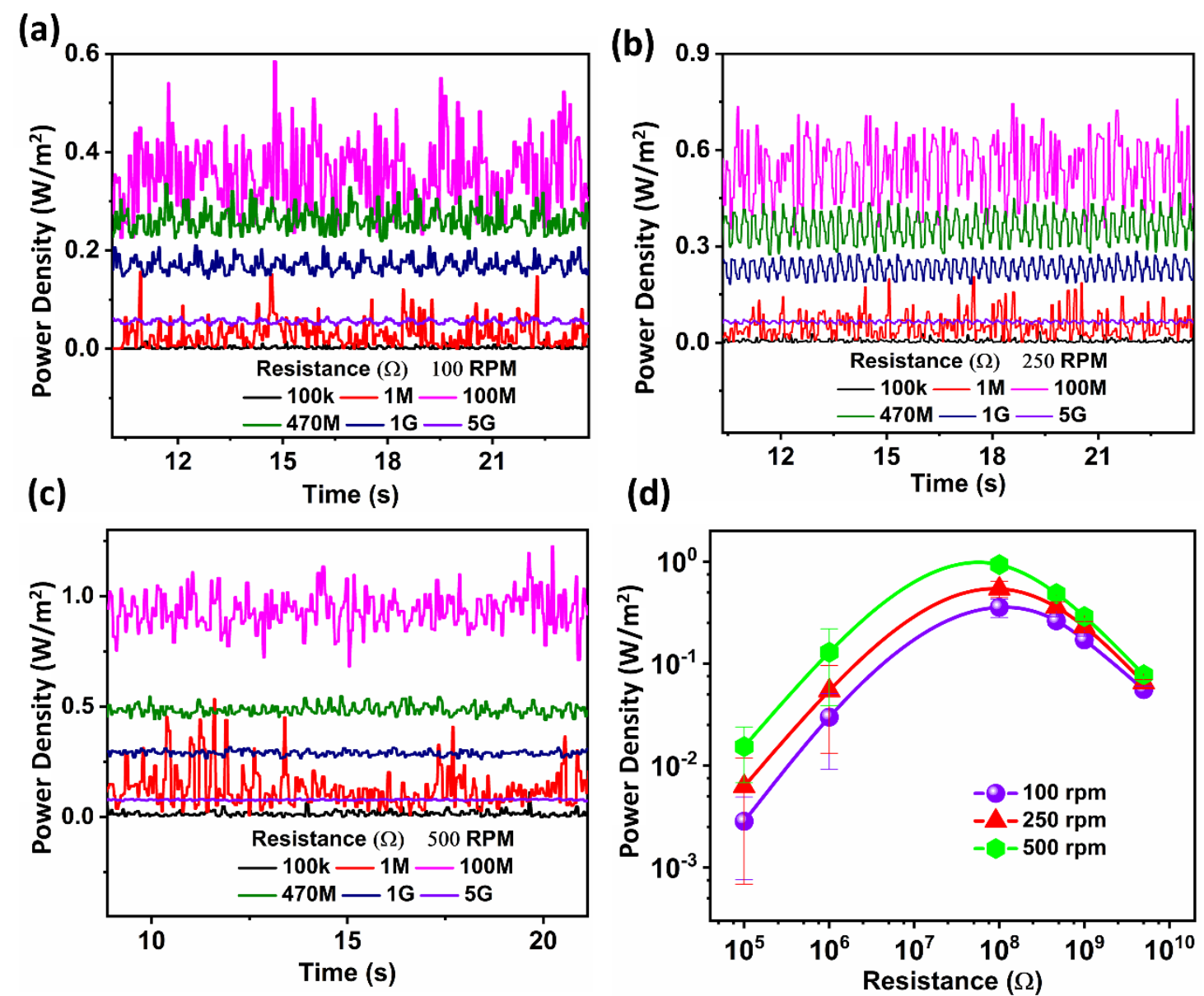

(e)

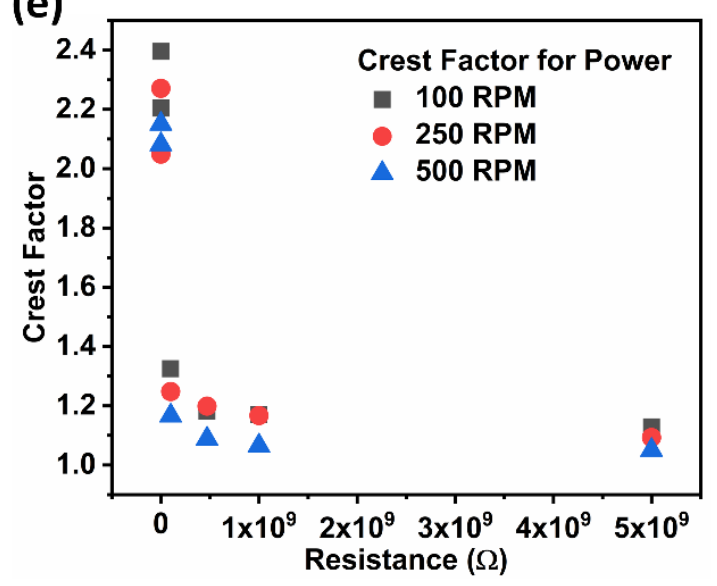

Figure 5: The output power (per unit area) for the asymmetric TENG architecture across increasing load resistances plotted against time, at a rotor speed of (a) 100 RPM, (b) 250 RPM, and, (c) 500 RPM. (d) The average output power corresponding to (a), (b) and (c), plotted 


\section{(a)}
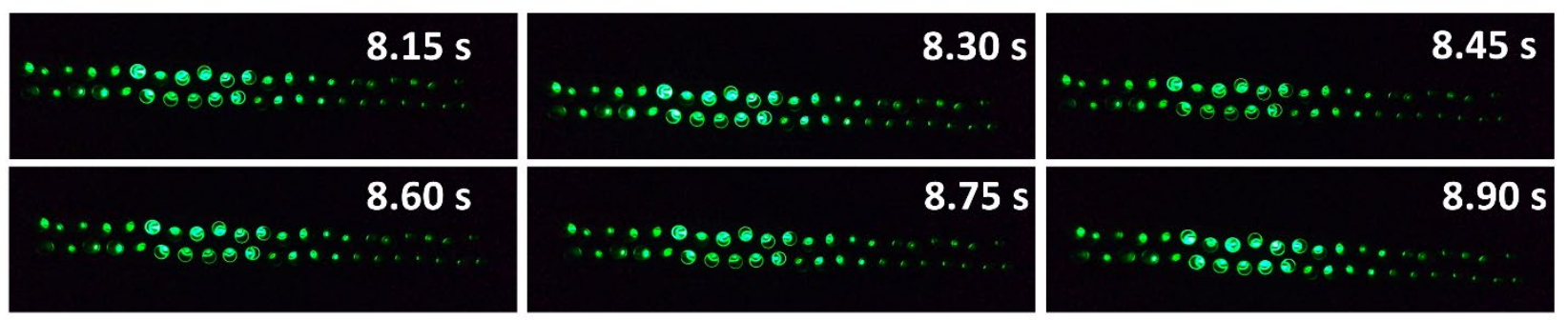

against increasing load, where the error bars indicate the standard deviation of 400 readings. (e) The crest factor for power outputs of the asymmetric TENG, plotted against increasing loads.

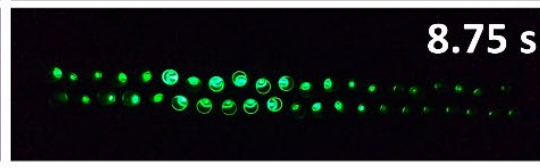

$\because \cdots, \ldots \theta 0000 .$.

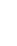

(b)

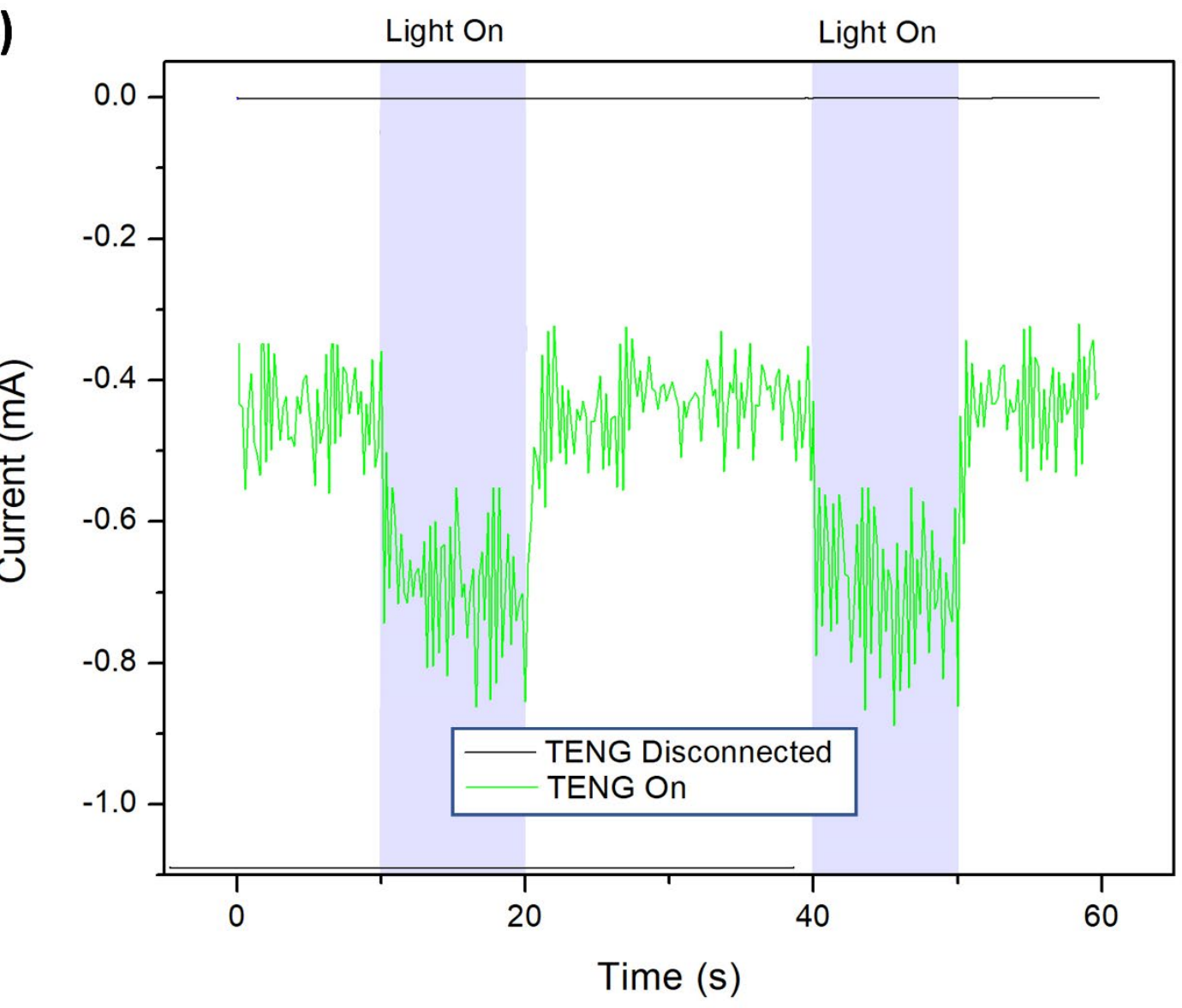


(a)

Oscillating arms for

exciting the TENGs

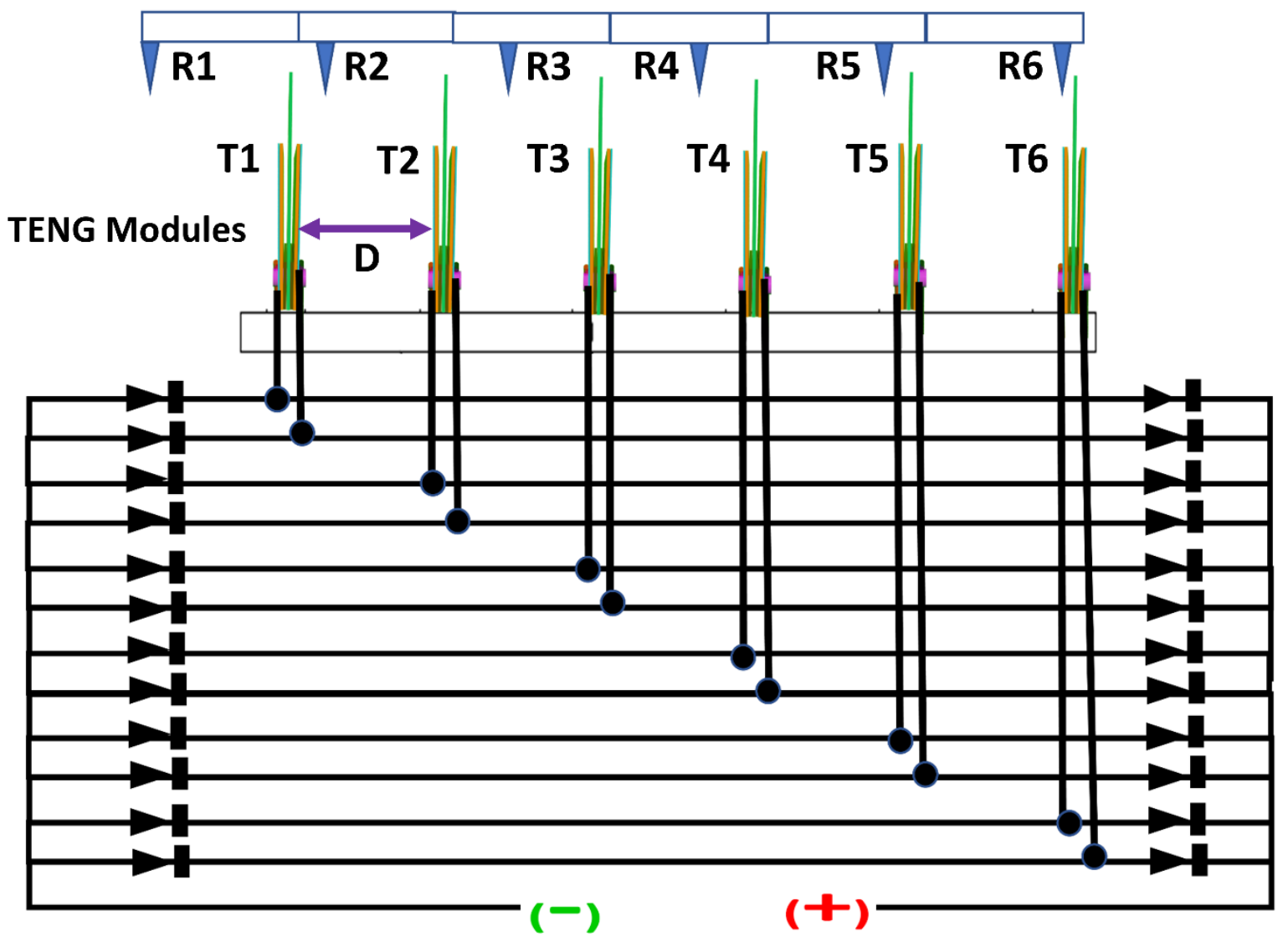

(b)

(c)
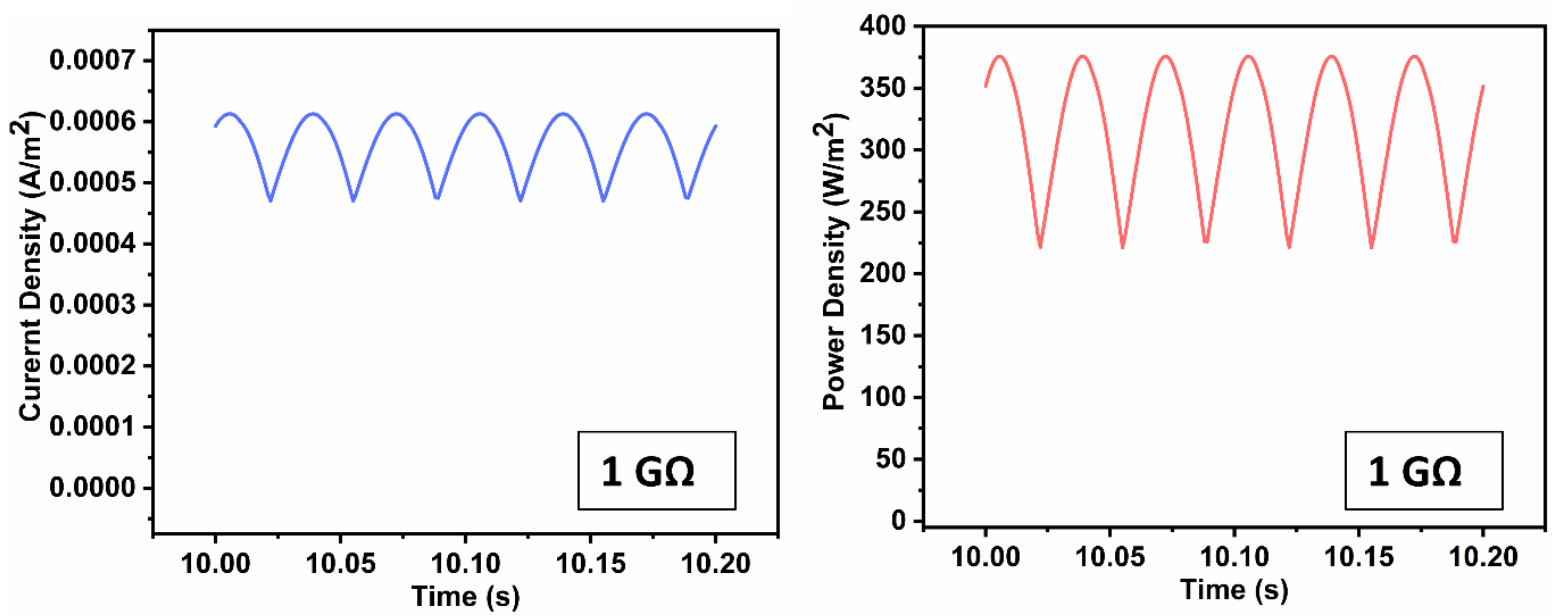

Figure 7: (a) Schematic of the DC TENG design compatible with the linear oscillating movements, and, the corresponding (b) current density, (c) power density outputs under an oscillation movement of $5 \mathrm{~mm}$ amplitude and $5 \mathrm{~Hz}$ frequency. 


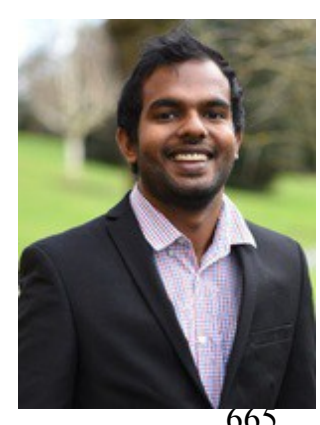

Dr Ishara Dharmasena is a Doctoral Prize Fellow at Wolfson School of Mechanical, Electrical and Manufacturing Engineering, Loughborough University. He received his B.Sc. (Engineering) degree from University of Moratuwa, Sri Lanka (2014), and, PhD degree at the Advanced Technology Institute (ATI), University of Surrey, specializing in energy harvesting and wearable electronics (2019). He has previously worked in the ATI as a research fellow, and, at Sri Lanka Institute of Nanotechnology (SLINTEC) as a research scientist. His research interests include mechanical energy harvesting, triboelectric nanogenerators, wearable electronics and flexible electronics.

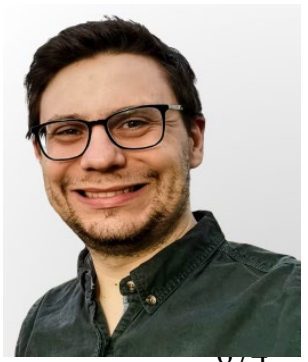

Dr. Harry Cronin received an MSci in Physics from Imperial College London in 2010, and an Engineering Doctorate (EngD) in Advanced Materials from the University of Surrey in 2018. His research interests have included perovskite photovoltaics, printable electronic materials, and 2D materials. He has held several positions within the defence and printed electronics industries.

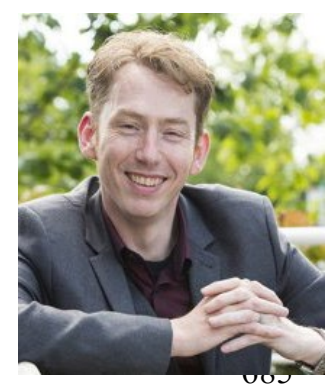

Prof. Robert Dorey holds the chair in Nanomaterials at the University of Surrey and is Fellow of the Institute Materials, Mining and Minerals (FIMMM) and Higher Education Academy (FHEA) as well as a Chartered Scientist and Engineer. Professor Dorey joined the University of Surrey from Cranfield University in 2014. Between 2003 and 2008 he held a prestigious Royal Academy of Engineering/EPSRC Research Fellowship. His research interests are focused on the synthesis \& manipulation of nanomaterials, through sustainable processing routes, for the production of functional devices for energy \& environmental applications.

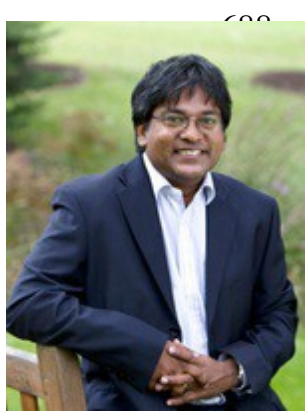

Prof. S.R.P. Silva is the Director of the Advanced Technology Institute at University of Surrey. He joined Surrey in 1995 after completing undergraduate and postgraduate degrees at Cambridge University. His research interests include nanoelectronics, largearea electronics and renewables, and his research outputs resulted in over 550 journal papers and 30 patents. He has over 19,000 citations, with an $\mathrm{H}$-index of 69, and, won research funding in excess of $£ 30 \mathrm{~m}$. He is a Fellow of the Royal Academy of Engineering, UK and a Fellow of the National Academy of Sciences Sri Lanka. 


\section{Supporting Information}

Direct Current Contact-Mode Triboelectric Nanogenerators via Systematic Phase Shifting

R D I G Dharmasena, H M Cronin, $R$ A Dorey, S R P Silva* 


\section{Supplementary Note 1: Device parameters used in the Theory section}

TENG Layer 1

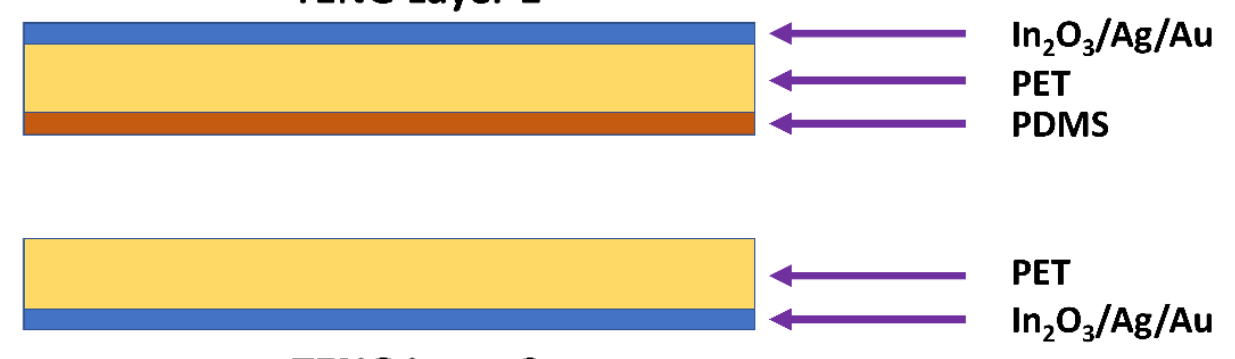

TENG Layer 2

Figure S1: The structure and the materials of the basic TENG device, which was utilised for the simulations and the experimental results of the theory section of the main text.

A simple VCPTENG architecture similar to that used in our previous work ${ }^{[18]}$, was used in the simulations and the experimental results of the Theory section. Both the TENG layers were composed of two $\mathrm{In}_{2} \mathrm{O}_{3}$ electrodes, and polyethylene terephthalate (PET) sheets. TENG layer 1 consisted of a polydimethylsiloxane (PDMS) contact surface. Dimensions of the TENG layers are as follows:

Length of TENG layers $=5 \mathrm{~cm}$

Width of TENG layers $=5 \mathrm{~cm}$

Thickness of PET $=200 \mu \mathrm{m}$

Thickness of PDMS $=20 \mu \mathrm{m}$

Dielectric constant of TENG layer $1=3.24$

Dielectric constant of TENG layer $2=3.3$ 


\section{Supplementary Note 2: Current and power against extended number of TENG units}

(a)

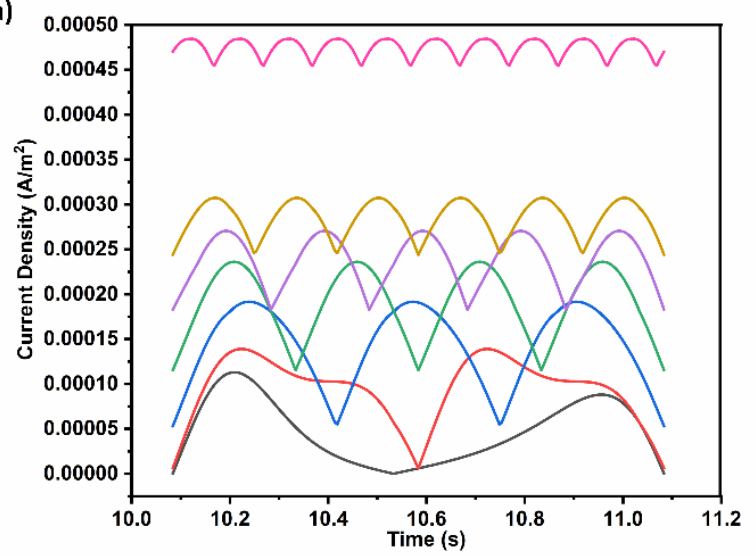

(b)

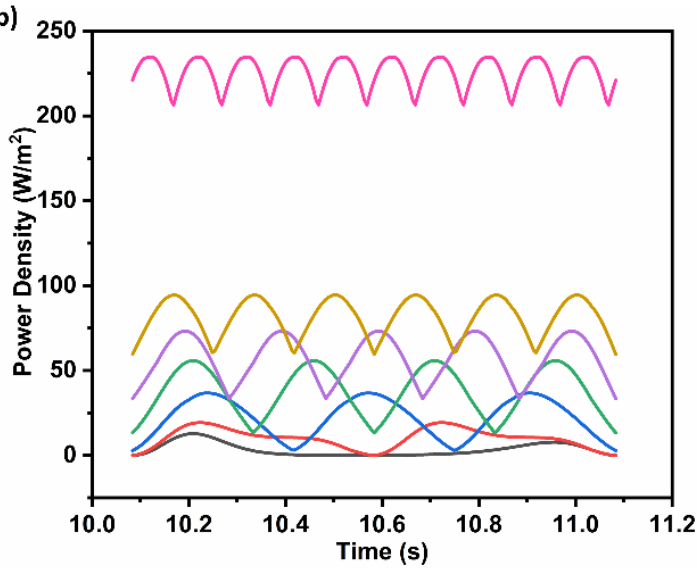

$$
-1 \text { unit }-2 \text { units }-3 \text { units }-4 \text { units }-5 \text { units }-6 \text { units }-10 \text { units }
$$

Figure S2: DDEF model simulations for the variation of (a) current density, and, (b) power density through a 1 G $\Omega$ load, subjected to a $1 \mathrm{~mm}$ amplitude and $1 \mathrm{~Hz}$ frequency motion profile similar to Fig. 2, extended to demonstrate the case for 10 TENG units.

Fig. S2 demonstrates the case of the use of an extended number of TENG units (poles) with systematic phase shifting, and its effect on the current density (Fig. S2a) and the power density (Fig. S2b). Compared to the cases considered in the manuscript (1 to 6 TENG units), the extended case with 10 TENG units provide higher output performances, along with better DC characteristics resulting in a current crest factor of 1.021 and power crest factor of 1.042. This further supports the fact that increasing the number of TENG poles results in the resultant overall output being higher in magnitude as well as being closer to an ideal DC waveform with crest factors close to 1 . 